\title{
Efficient Reduced Basis Methods for Saddle Point Problems With Applications in Groundwater Flow
}

DOI:

10.1137/16M1108856

\section{Document Version}

Accepted author manuscript

Link to publication record in Manchester Research Explorer

\section{Citation for published version (APA):}

Newsum, C., \& Powell, C. (2017). Efficient Reduced Basis Methods for Saddle Point Problems With Applications in Groundwater Flow. SIAM / ASA Journal on Uncertainty Quantification , 5(1). https://doi.org/10.1137/16M1108856

\section{Published in:}

SIAM / ASA Journal on Uncertainty Quantification

\section{Citing this paper}

Please note that where the full-text provided on Manchester Research Explorer is the Author Accepted Manuscript or Proof version this may differ from the final Published version. If citing, it is advised that you check and use the publisher's definitive version.

\section{General rights}

Copyright and moral rights for the publications made accessible in the Research Explorer are retained by the authors and/or other copyright owners and it is a condition of accessing publications that users recognise and abide by the legal requirements associated with these rights.

\section{Takedown policy}

If you believe that this document breaches copyright please refer to the University of Manchester's Takedown Procedures [http://man.ac.uk/04Y6Bo] or contact uml.scholarlycommunications@manchester.ac.uk providing relevant details, so we can investigate your claim.

\section{OPEN ACCESS}




\title{
EFFICIENT REDUCED BASIS METHODS FOR SADDLE POINT PROBLEMS WITH APPLICATIONS IN GROUNDWATER FLOW
}

\author{
CRAIG J. NEWSUM* AND CATHERINE E. POWELL ${ }^{\dagger}$
}

\begin{abstract}
Reduced basis methods (RBMs) are recommended to reduce the computational cost of solving parameter-dependent PDEs in scenarios where many choices of parameters need to be considered, for example in uncertainty quantification (UQ). A reduced basis is constructed during a computationally demanding offline (or setup) stage that allows the user to obtain cheap approximations for parameters choices of interest, online. In this paper we consider RBMs for parameter-dependent saddle point problems, in particular the one that arises in the mixed formulation of the Darcy flow problem in groundwater flow modelling. We apply a discrete empirical interpolation method (DEIM) to approximate the inverse of the diffusion coefficient, which depends non-affinely on the system parameters. We develop an efficient RBM that exploits the DEIM approximation and combine it with a sparse grid stochastic collocation mixed finite element method (SCMFEM) to construct a surrogate solution, which then allows for efficient forward UQ. Through numerical experiments we demonstrate that significant computational savings can be made when we use the RB-DEIM-SCMFEM scheme over standard high fidelity methods. For groundwater flow problems, we provide a thorough cost assessment of the new method and show how the size of the reduced basis, and hence, the extent of the savings, depends on the statistical properties of the input parameters.
\end{abstract}

Key words. reduced basis methods, saddle point problems, stochastic collocation, uncertainty quantification

AMS subject classifications. $35 \mathrm{R} 60,60 \mathrm{H} 35,65 \mathrm{~N} 22,65 \mathrm{~N} 30$

1. Introduction. We focus on the efficient numerical solution of parameter-dependent saddle point problems with the following abstract form. Let $D \subset \mathbb{R}^{d}$ be a given bounded spatial domain and let $V$ and $Q$ be Hilbert spaces on $D$, with inner products $\langle\cdot, \cdot\rangle_{V},\langle\cdot, \cdot\rangle_{Q}$ and induced norms $\|\cdot\|_{V},\|\cdot\|_{Q}$. In addition, let $\Gamma \subset \mathbb{R}^{M}$ be an $M$-dimensional parameter space. Given $\mathbf{y} \in \Gamma$, we want to find $(\vec{u}(\cdot, \mathbf{y}), p(\cdot, \mathbf{y})) \in V \times Q$ satisfying

$$
\begin{array}{rlrl}
a(\vec{u}(\cdot, \mathbf{y}), \vec{v}, \mathbf{y})+b(\vec{v}, p(\cdot, \mathbf{y}), \mathbf{y}) & =l(\vec{v}), & & \forall \vec{v} \in V, \\
b(\vec{u}(\cdot, \mathbf{y}), q, \mathbf{y}) & =m(q), & \forall q \in Q .
\end{array}
$$

Weak formulations of many well-known systems of PDEs give rise to saddle point problems of the form (1.1). For example, the Stokes equations, the steady-state Navier-Stokes equations, and mixed formulations of the Darcy flow problem, in situations where the coefficients and/or the spatial domain are represented as functions of parameters $\mathbf{y} \in \Gamma$. For a fixed $\mathbf{y}=\left(y_{1}, \ldots, y_{M}\right) \in \Gamma$, the solution $(\vec{u}(\cdot, \mathbf{y}), p(\cdot, \mathbf{y}))$ can be approximated using standard techniques such as mixed finite element methods (so-called high fidelity methods).

In many complex applications (e.g., fluid flow modelling), obtaining a high fidelity approximation for even a single choice of $\mathbf{y}$ may take several hours or days of computation time. If real-time simulations are required for many $\mathbf{y} \in \Gamma$, then using high fidelity methods is infeasible. To avoid this computational burden, reduced basis methods (RBMs), see [31, 39], have been developed. The idea is to project the high fidelity problem with dimension $N_{h}$ (corresponding, say, to the number of degrees of freedom in a finite element mesh) onto a lower-dimensional subspace with dimension $N_{R}$. When $N_{R} \ll N_{h}$, we expect that the cost of solving the reduced problem will be significantly cheaper, for each $\mathbf{y} \in \Gamma$ of interest. Crucial to the success of RBMs, is that $N_{R}$ is 'not too large', which requires that the underlying solution manifold (as a function of $\mathbf{y}$ ) is smooth. There is a large literature on RBMs for scalar elliptic PDEs. However, saddle point problems are more challenging as two compatible reduced spaces are required. RBMs for Stokes flow with

\footnotetext{
${ }^{*}$ School of Mathematics, The University of Manchester, Oxford Road, Manchester, M13 9PL, UK (craig.newsum@postgrad.manchester.ac.uk).

${ }^{\dagger}$ School of Mathematics, The University of Manchester, Oxford Road, Manchester, M13 9PL, UK (c.powell@manchester.ac.uk).
} 
parameter-dependent viscosity and spatial domains have been well studied, see [24, 41, 42], and RBMs for Stokes flow coupled with the elliptic formulation of Darcy flow are studied in [34].

RBMs separate the computational work into two stages, an expensive offline stage where we carry out tasks whose costs depend on $N_{h}$, and a (hopefully) cheaper online stage where we only perform tasks whose costs depend on $N_{R}$. It is conventional to think of the offline stage as a set-up phase, carried out once ahead of the main numerical task. To facilitate this offline-online separation, it is essential that we can separate the dependence of the system inputs on $\mathbf{y} \in \Gamma$ from their dependence on $\mathbf{x} \in D$. Thus, in (1.1), we require that

$$
a(\cdot, \cdot, \mathbf{y})=\sum_{k=1}^{K} \theta_{k}(\mathbf{y}) a_{k}(\cdot, \cdot),
$$

where $a_{k}: V \times V \rightarrow \mathbb{R}$ is a parameter-independent bilinear form and $\theta_{k}(\mathbf{y})$ is a function of $\mathbf{y}$, and similarly for $b(\cdot, \cdot, \mathbf{y})$. This allows us to precompute quantities that do not depend on $\mathbf{y}$ offline. Provided that $K \ll N_{h}$, we can assemble and solve a reduced version of (1.1) online, at a cost that is independent of $N_{h}$. When the inputs have non-affine parameter dependence, we can use empirical interpolation methods [3, 12, 21, 28] to generate approximations of the form (1.2). This introduces an error, which must be controlled, and balanced against the reduced basis error.

In this paper we focus on the application of RBMs to the numerical solution of systems of PDEs with uncertain inputs, whose weak formulations give rise to saddle point problems of the form (1.1). If we represent the uncertain inputs as functions of $M$ random variables (e.g. using a Karhunen-Loève expansion, see [30]), whose image is the parameter space $\Gamma \subset \mathbb{R}^{M}$, then choosing a particular $\mathbf{y} \in \Gamma$ corresponds to generating one sample of the inputs. Our goal is to propagate the uncertainty in the inputs through the system and approximate statistical quantities of interest (QoIs), for example the mean and variance of the solution. This is known as forward uncertainty quantification (UQ), see [45, 48]. The simplest way to perform forward UQ is to use sampling methods, which require repeated solution of (1.1) for many choices of $\mathbf{y}$. RBMs offer a promising way to speed up forward UQ assessments when sampling methods are used, see [10, 14, 15, 22]. They have also been applied to inverse problems [47, 17], and optimal control problems [16].

The two most commonly used sampling methods for forward UQ, see [29], are Monte Carlo (MC) and stochastic collocation methods (SCMs). The former are simple to implement but only allow for the approximation of moments. SCMs, on the other hand, provide an interpolant in both physical and parameter space. This can be used to approximate statistical moments but also acts as a surrogate that can be used to find approximations to $\vec{u}(\cdot, \mathbf{y})$ and $p(\cdot, \mathbf{y})$ for further choices of $\mathbf{y} \in \Gamma$ (in design or control experiments) and to approximate other QoIs. SCMs were first applied to scalar elliptic PDEs in $[1,35,50]$ and have since been applied to many other problems, such as the Darcy flow model $[23,25]$, in a high-fidelity setting. SCMs often converge much faster than standard MC methods (whose error is $\mathcal{O}\left(N^{-1 / 2}\right)$, where $N$ is the number of samples). The rate of convergence of $\mathrm{MC}$, however, is independent of the number $M$ of parameters. Alternative methods have been proposed for dealing with high-dimensional problems and these can also be combined with RBMs to reduce costs. Two such examples are the ANOVA decomposition which decomposes the high-dimensional problem into low-dimensional problems, see [32], and adaptive sparse grid collocation [13]. Finally, we mention the stochastic Galerkin method [2], which is not a sampling method. This leads to a single large linear system that requires specialised solvers, see for example [37]. In this paper we develop an efficient RBM and combine it with a sparse grid stochastic collocation mixed finite element method (SCMFEM) for a class of parameter-dependent saddle point problems. A similar strategy has been employed in $[5,15,22]$ for scalar elliptic PDEs.

In Section 2 we describe the reduced formulation of general parameter-dependent saddle point problems of the form (1.1). We also review sufficient conditions for the well-posedness of both high fidelity and reduced mixed finite element approximations. In Section 3 we introduce the Darcy 
flow model as a special case, and then derive the linear systems that arise from the high fidelity and reduced formulations. In Section 4 we develop a reduced basis method which uses a nonstandard training technique to construct a compatible pair of reduced approximation spaces in an offline stage, as well as a so-called DEIM approximation to the inverse of the diffusion coefficient. In Section 5 we describe a sparse grid SCMFEM which we then combine with the RB method and DEIM approximation. Finally, in Section 6 we present numerical experiments to demonstrate the superior performance of the new RB-DEIM-SCMFEM scheme, over the standard high fidelity SCMFEM scheme, for performing forward UQ in groundwater flow applications.

2. Parameter-dependent saddle point problems. We begin by considering the reduced formulation of general parameter-dependent saddle point problems of the form (1.1). The theoretical framework presented here is standard (e.g., see [9] and [24]).

We assume that for each $\mathbf{y} \in \Gamma, a(\cdot, \cdot, \mathbf{y}): V \times V \rightarrow \mathbb{R}$ and $b(\cdot, \cdot, \mathbf{y}): V \times Q \rightarrow \mathbb{R}$ are bounded bilinear forms and $l: V \rightarrow \mathbb{R}$ and $m: Q \rightarrow \mathbb{R}$ are also bounded. Now, for each $\mathbf{y} \in \Gamma$ we assume that $a(\cdot, \cdot, \mathbf{y})$ is coercive over

$$
V_{0}=\{\vec{v} \in V: b(\vec{v}, q, \mathbf{y})=0, \quad \forall q \in Q\} \subset V,
$$

the nullspace of $V$. That is,

$$
\alpha(\mathbf{y}):=\inf _{\vec{v} \in V_{0}} \frac{a(\vec{v}, \vec{v}, \mathbf{y})}{\|\vec{v}\|_{V}^{2}}>0 .
$$

We also assume that for each $\mathbf{y} \in \Gamma, b(\cdot, \cdot, \mathbf{y})$ satisfies an inf-sup condition. Specifically,

$$
\beta(\mathbf{y}):=\inf _{q \in Q} \sup _{\vec{v} \in V} \frac{b(\vec{v}, q, \mathbf{y})}{\|\vec{v}\|_{V}\|q\|_{Q}}>0 .
$$

These four conditions are sufficient to guarantee that (1.1) is well-posed for a fixed $\mathbf{y} \in \Gamma$, see [9].

Next, we introduce finite-dimensional approximation spaces $V_{h} \subset V$ and $Q_{h} \subset Q$, and consider the high fidelity problem: given $\mathbf{y} \in \Gamma$, find $\left(\vec{u}_{h}(\cdot, \mathbf{y}), p_{h}(\cdot, \mathbf{y})\right) \in V_{h} \times Q_{h}$ satisfying

$$
\begin{array}{rlrl}
a\left(\vec{u}_{h}(\cdot, \mathbf{y}), \vec{v}_{h}, \mathbf{y}\right)+b\left(\vec{v}_{h}, p_{h}(\cdot, \mathbf{y}), \mathbf{y}\right) & =l\left(\vec{v}_{h}\right), & & \forall \vec{v}_{h} \in V_{h}, \\
b\left(\vec{u}_{h}(\cdot, \mathbf{y}), q_{h}, \mathbf{y}\right) & =m\left(q_{h}\right), \quad & \forall q_{h} \in Q_{h} .
\end{array}
$$

In this work, we assume that $V_{h}$ and $Q_{h}$ are finite element spaces, so that $h$ denotes the characteristic mesh size. In the language of RBMs, we call $\left(\vec{u}_{h}(\cdot, \mathbf{y}), p_{h}(\cdot, \mathbf{y})\right)$ a snapshot pair. To ensure well-posedness of the high fidelity problem, we assume that

$$
\alpha_{h}(\mathbf{y}):=\inf _{\vec{v}_{h} \in V_{0, h}} \frac{a\left(\vec{v}_{h}, \vec{v}_{h}, \mathbf{y}\right)}{\left\|\vec{v}_{h}\right\|_{V}^{2}}>0,
$$

where

$$
V_{0, h}=\left\{\vec{v}_{h} \in V_{h}: b\left(\vec{v}_{h}, q_{h}, \mathbf{y}\right)=0, \quad \forall q_{h} \in Q_{h}\right\} \subset V_{h},
$$

is the nullspace of $V_{h}$, and secondly that

$$
\beta_{h}(\mathbf{y}):=\inf _{q_{h} \in Q_{h}} \sup _{\vec{v}_{h} \in V_{h}} \frac{b\left(\vec{v}_{h}, q_{h}, \mathbf{y}\right)}{\left\|\vec{v}_{h}\right\|_{V}\left\|q_{h}\right\|_{Q}}>0 .
$$

In particular, this means that $\alpha_{h}(\mathbf{y})$ and $\beta_{h}(\mathbf{y})$ must be bounded away from zero, independently of $h$. Note that neither of these conditions is satisfied automatically. The spaces $V_{h}$ and $Q_{h}$ must be compatible for the problem at hand. 
Now, let $V_{R} \subset V_{h}$ and $Q_{R} \subset Q_{h}$ denote reduced spaces, of smaller dimension than $V_{h}$ and $Q_{h}$, respectively. The reduced problem is: given $\mathbf{y} \in \Gamma$, find $\left(\vec{u}_{R}(\cdot, \mathbf{y}), p_{R}(\cdot, \mathbf{y})\right) \in V_{R} \times Q_{R}$ satisfying

$$
\begin{aligned}
a\left(\vec{u}_{R}(\cdot, \mathbf{y}), \vec{v}_{R}, \mathbf{y}\right)+b\left(\vec{v}_{R}, p_{R}(\cdot, \mathbf{y}), \mathbf{y}\right) & =l\left(\vec{v}_{R}\right), & & \forall \vec{v}_{R} \in V_{R}, \\
b\left(\vec{u}_{R}(\cdot, \mathbf{y}), q_{R}, \mathbf{y}\right) & =m\left(q_{R}\right), & & \forall q_{R} \in Q_{R} .
\end{aligned}
$$

If the analogues of conditions (2.5) and (2.7) are satisfied, then (2.8) is well-posed. We need

$$
\alpha_{R}(\mathbf{y}):=\inf _{\vec{v}_{R} \in V_{0, R}} \frac{a\left(\vec{v}_{R}, \vec{v}_{R}, \mathbf{y}\right)}{\left\|\vec{v}_{R}\right\|_{V}^{2}}>0
$$

where

$$
V_{0, R}=\left\{\vec{v}_{R} \in V_{R}: b\left(\vec{v}_{R}, q_{R}, \mathbf{y}\right)=0, \quad \forall q_{R} \in Q_{R}\right\} \subset V_{R},
$$

is the nullspace of $V_{R}$, and secondly that

$$
\beta_{R}(\mathbf{y}):=\inf _{q_{R} \in Q_{R}} \sup _{\vec{v}_{R} \in V_{R}} \frac{b\left(\vec{v}_{R}, q_{R}, \mathbf{y}\right)}{\left\|\vec{v}_{R}\right\|_{V}\left\|q_{R}\right\|_{Q}}>0 .
$$

Reduced saddle point problems are challenging as (2.9) and (2.11) are not automatically satisfied for arbitrary choices of $V_{R}$ and $Q_{R}$, even if $V_{R} \subset V_{h}, Q_{R} \subset Q_{h}$, and $V_{h}$ and $Q_{h}$ are chosen so that (2.4) is well-posed. It is an open question what is the 'best' choice of $V_{R}$ and $Q_{R}$ to ensure that the reduced basis errors $\left\|\vec{u}_{h}(\cdot, \mathbf{y})-\vec{u}_{R}(\cdot, \mathbf{y})\right\|_{V}$ and $\left\|p_{h}(\cdot, \mathbf{y})-p_{R}(\cdot, \mathbf{y})\right\|_{Q}$ decay to zero as quickly as possible, as $N_{R}$ increases. Some possibilities for Stokes problems are discussed in [24].

If (2.9) and (2.11) are satisfied, we immediately obtain bounds for the reduced basis error, similar to standard high fidelity results (see [24]). Let $a_{\max }(\mathbf{y})$ and $b_{\max }(\mathbf{y})$ denote the boundedness constants for $a(\cdot, \cdot, \mathbf{y})$ and $b(\cdot, \cdot, \mathbf{y})$, respectively. Then, for a given $\mathbf{y} \in \Gamma$ we have

$$
\begin{aligned}
\left\|\vec{u}_{h}(\cdot, \mathbf{y})-\vec{u}_{R}(\cdot, \mathbf{y})\right\|_{V} \leq & \left(1+\frac{a_{\max }(\mathbf{y})}{\alpha_{R}(\mathbf{y})}\right) \inf _{v_{R} \in V_{0, R}}\left\|\vec{u}_{h}(\cdot, \mathbf{y})-\vec{v}_{R}(\cdot, \mathbf{y})\right\|_{V} \\
& +\left(\frac{1}{\alpha_{R}(\mathbf{y})}\right) \inf _{q_{R} \in Q_{R}}\left\|p_{h}(\cdot, \mathbf{y})-q_{R}(\cdot, \mathbf{y})\right\|_{Q},
\end{aligned}
$$

c.f. [11, Theorem 12.3.7], and

$$
\begin{aligned}
\left\|p_{h}(\cdot, \mathbf{y})-p_{R}(\cdot, \mathbf{y})\right\|_{Q} \leq & \left(\frac{a_{\max }(\mathbf{y})}{\alpha_{R}(\mathbf{y})}\right)\left\|\vec{u}_{h}(\cdot, \mathbf{y})-\vec{u}_{R}(\cdot, \mathbf{y})\right\|_{V} \\
& +\left(1+\frac{1}{\beta_{R}(\mathbf{y})}\right) \inf _{q_{R} \in Q_{R}}\left\|p_{h}(\cdot, \mathbf{y})-q_{R}(\cdot, \mathbf{y})\right\|_{Q}
\end{aligned}
$$

c.f. [11, Theorem 12.5.12]. Thus, if $\alpha_{R}(\mathbf{y}) \rightarrow 0$ or $\beta_{R}(\mathbf{y}) \rightarrow 0$ as $N_{R} \rightarrow \infty$, the method is unstable. A simple approach is to construct the reduced spaces from snapshots (solutions to the high fidelity problem). Suppose we take $\Theta=\left\{\mathbf{y}_{1}, \ldots, \mathbf{y}_{N_{R}}\right\}$, a set of $N_{R}$ points in the parameter domain, and the set of snapshot pairs $\left\{\left(\vec{u}_{h}\left(\cdot, \mathbf{y}_{i}\right), p_{h}\left(\cdot, \mathbf{y}_{i}\right)\right), i=1, \ldots, N_{R}\right\}$. Then, we could choose

$$
V_{R}:=\operatorname{span}\left\{\vec{u}_{h}\left(\cdot, \mathbf{y}_{i}\right)\right\}_{i=1}^{N_{R}}, \quad Q_{R}:=\operatorname{span}\left\{p_{h}\left(\cdot, \mathbf{y}_{i}\right)\right\}_{i=1}^{N_{R}} .
$$

As established in $[24,41,42]$, however, this does not provide an inf-sup stable pair for (2.8). In particular, $V_{R}$ is not rich enough for (2.11) to hold. Indeed, it well known from deterministic mixed finite element analysis, that the spaces should not have the same dimension; $V_{R}$ must be augmented. Following the approach introduced in [42], we define an operator $T: Q_{h} \rightarrow V_{h}$ via

$$
\langle T q, \vec{v}\rangle_{V}=b(\vec{v}, q, \mathbf{y}), \quad \forall \vec{v} \in V_{h} .
$$


The functions $T q$ are called supremizer functions since, given $q \in Q_{h}$, we have

$$
T q=\arg \sup _{\vec{v} \in V_{h}} \frac{b(\vec{v}, q, \mathbf{y})}{\|\vec{v}\|_{V}} .
$$

Instead of using (2.14), we choose the reduced spaces as

$$
V_{R}:=\operatorname{span}\left\{\vec{u}_{h}\left(\cdot, \mathbf{y}_{i}\right), T p_{h}\left(\cdot, \mathbf{y}_{i}\right)\right\}_{i=1}^{N_{R}}, \quad Q_{R}:=\operatorname{span}\left\{p_{h}\left(\cdot, \mathbf{y}_{i}\right)\right\}_{i=1}^{N_{R}},
$$

so that $\operatorname{dim}\left(V_{R}\right)=2 \times \operatorname{dim}\left(Q_{R}\right)=2 \times N_{R}$. With this choice, it can be shown that (2.11) is satisfied. Indeed, for all $\mathbf{y} \in \Gamma, \beta_{R}(\mathbf{y}) \geq \beta_{h}(\mathbf{y})$ (see [42]), so as long as $\beta_{h}(\mathbf{y})$ does not decay to zero as $h \rightarrow 0$ ( $V_{h}$ and $Q_{h}$ are compatible), then neither will $\beta_{R}(\mathbf{y})$, as $N_{R}$ increases.

Let us now briefly consider (2.9). If, for the high fidelity problem $(2.4), a(\cdot, \cdot, \mathbf{y})$ is coercive on the whole of $V_{h}$ (or even $V$ ) rather than just on a strict subset $V_{0, h}$, then (2.9) follows immediately. That is, coercivity on $V_{h}$ in $\|\cdot\|_{V}$ automatically gives coercivity on $V_{0, R}$ since $V_{0, R} \subset V_{R} \subset V_{h} \subset V$. For example, this is the case for Stokes equations, see [24, 41, 42]. In the problem we study below, the continuous and high-fidelity problems are only coercive with respect to $\|\cdot\|_{V}$ on $V_{0}$ and $V_{0, h}$, respectively. This does not cause a problem with well-posedness of (2.8), but the a priori error estimate (2.12) is not automatically obtained from the standard theory.

3. Darcy flow model. We now consider the Darcy flow problem, a system of PDEs that arises when modelling fluid flow through a porous medium. Typically, the permeability (or diffusion) coefficients are not known at every spatial location $\mathrm{x} \in D$ (epistemic uncertainty). Moreover, there may be measurement errors (aleatoric uncertainty). In such situations, it is common to model the unknown coefficient function as a random field $a(\mathbf{x}, \omega)$.

Let $\mathcal{D} \subset \mathbb{R}^{2}$ be a bounded physical domain whose boundary $\partial \mathcal{D}$ is decomposed into two disjoint subsets so that $\partial \mathcal{D}=\partial \mathcal{D}_{\mathrm{D}} \cup \partial \mathcal{D}_{\mathrm{N}}$. In addition, let $(\Omega, \mathcal{F}, \mathbb{P})$ be a probability space, where $\Omega$ denotes a sample space, $\mathcal{F}$ is a sigma algebra and $\mathbb{P}$ is a probability measure. We want to find a velocity field $\vec{u}: \mathcal{D} \times \Omega \rightarrow \mathbb{R}^{2}$ and a pressure field $p: \mathcal{D} \times \Omega \rightarrow \mathbb{R}$ such that $\mathbb{P}$-a.s.,

$$
\begin{aligned}
a^{-1}(\mathbf{x}, \omega) \vec{u}(\mathbf{x}, \omega)+\nabla p(\mathbf{x}, \omega) & =0, & & \mathbf{x} \in \mathcal{D}, \\
\nabla \cdot \vec{u}(\mathbf{x}, \omega) & =f(\mathbf{x}), & & \mathbf{x} \in \mathcal{D}, \\
p(\mathbf{x}, \omega) & =g(\mathbf{x}), & & \mathbf{x} \in \partial \mathcal{D}_{\mathrm{D}}, \\
\vec{u}(\mathbf{x}, \omega) \cdot \vec{n} & =0, & & \mathbf{x} \in \partial \mathcal{D}_{\mathrm{N}} .
\end{aligned}
$$

We assume that $a(\mathbf{x}, \omega)$ is second-order and there exist constants $a_{\min }$ and $a_{\max }$ such that

$$
0 \leq a_{\min } \leq a^{-1}(\mathbf{x}, \omega) \leq a_{\max }<\infty, \quad \text { a.e. in } \mathcal{D} \times \Omega .
$$

The first equation in (3.1) is Darcy's law $\vec{u}=-a \nabla p$, relating the velocity to the pressure gradient and the second equation relates the fluid mass to source and sink terms. Although both equations can be combined to obtain a standard scalar elliptic PDE for the pressure, our aim is to propagate the uncertainty through the PDE model and accurately approximate both $p(\mathbf{x}, \omega)$ and $\vec{u}(\mathbf{x}, \omega)$.

If the mean $\mu(\mathbf{x})=\mathbb{E}[a(\mathbf{x}, \omega)]$ and covariance function $C\left(\mathbf{x}_{1}, \mathbf{x}_{2}\right)$ of $a(\mathbf{x}, \omega)$ are known, then $a(\mathbf{x}, \omega)$ can be approximated by a function of $M$ random variables $\xi_{k}: \Omega \rightarrow \mathbb{R}$, by using a truncated Karhunen-Loève (KL) expansion [33],

$$
a_{M}(\mathbf{x}, \boldsymbol{\xi}(\omega))=\mu(\mathbf{x})+\sum_{k=1}^{M} \sqrt{\lambda_{k}} \phi_{k}(\mathbf{x}) \xi_{k}(\omega) .
$$

Here, $\lambda_{k}$ and $\phi_{k}$ are eigenvalues and eigenfunctions of the integral operator associated with the covariance function, and we assume the $\xi_{k}$ are independent. Alternatively, we may have another 
underlying second-order random field $z(\mathbf{x}, \omega)$ with known mean and covariance, with $a(\mathbf{x}, \omega)=$ $\exp (z(\mathbf{x}, \omega))$. This is common in groundwater flow modelling (e.g., see [23, 26]). We may then compute an approximation $a_{M}(\mathbf{x}, \boldsymbol{\xi})=\exp \left(z_{M}(\mathbf{x}, \boldsymbol{\xi})\right)$, where $z_{M}(\mathbf{x}, \boldsymbol{\xi})$ is a truncated KL expansion. The important point is that the inverse of the diffusion coefficient is some function of a vector of independent random variables $\boldsymbol{\xi}=\left(\xi_{1}, \ldots, \xi_{M}\right)$.

Instead of working with the random variable $\boldsymbol{\xi}$, with joint probability density function $\rho(\mathbf{y})$, we may work in terms of a vector of parameters $\mathbf{y}=\left(y_{1}, \ldots, y_{M}\right)$, with $y_{k}=\xi_{k}(\Omega)$, which takes values in $\Gamma:=\boldsymbol{\xi}(\Omega) \subset \mathbb{R}^{M}$. If we want to approximate samples of the velocity and pressure fields, we can replace the stochastic problem (3.1) with an equivalent parameter-dependent deterministic one. We assume that for each $\mathbf{y} \in \Gamma$ there exist constants $a_{\min }(\mathbf{y})$ and $a_{\max }(\mathbf{y})$ such that

$$
0 \leq a_{\min }(\mathbf{y}) \leq a_{M}^{-1}(\mathbf{x}, \mathbf{y}) \leq a_{\max }(\mathbf{y})<\infty .
$$

Given $\mathbf{y} \in \Gamma$, we then want to find $\vec{u}(\cdot, \mathbf{y}): \mathcal{D} \rightarrow \mathbb{R}^{2}$ and $p(\cdot, \mathbf{y}): \mathcal{D} \rightarrow \mathbb{R}$ satisfying

$$
\begin{aligned}
a_{M}^{-1}(\mathbf{x}, \mathbf{y}) \vec{u}(\mathbf{x}, \mathbf{y})+\nabla p(\mathbf{x}, \mathbf{y}) & =0, & & \mathbf{x} \in \mathcal{D}, \\
\nabla \cdot \vec{u}(\mathbf{x}, \mathbf{y}) & =f(\mathbf{x}), & & \mathbf{x} \in \mathcal{D}, \\
p(\mathbf{x}, \mathbf{y}) & =g(\mathbf{x}), & & \mathbf{x} \in \partial \mathcal{D}_{\mathrm{D}}, \\
\vec{u}(\mathbf{x}, \mathbf{y}) \cdot \vec{n} & =0, & & \mathbf{x} \in \partial \mathcal{D}_{\mathrm{N}} .
\end{aligned}
$$

Now, let $Q=L^{2}(\mathcal{D})$ and $V=\mathrm{H}_{0, \mathrm{~N}}(\operatorname{div}, \mathcal{D})$, where

$$
\mathrm{H}_{0, \mathrm{~N}}(\operatorname{div}, \mathcal{D})=\left\{\vec{v}: \mathcal{D} \rightarrow \mathbb{R}^{2}: \vec{v} \in\left(L^{2}(\mathcal{D})\right)^{2}, \nabla \cdot \vec{v} \in L^{2}(\mathcal{D}), \vec{v} \cdot \vec{n}=0 \text { on } \partial \mathcal{D}_{\mathrm{N}}\right\}
$$

and define the norm

$$
\|\vec{u}\|_{\mathrm{H}(\operatorname{div}, \mathcal{D})}=\left(\|\vec{u}\|_{L^{2}(\mathcal{D})}^{2}+\|\nabla \cdot \vec{u}\|_{L^{2}(\mathcal{D})}^{2}\right)^{1 / 2} .
$$

For each $\mathbf{y} \in \Gamma$, the weak formulation of $(3.5)$ is: find $(\vec{u}(\cdot, \mathbf{y}), p(\cdot, \mathbf{y})) \in V \times Q$ satisfying

$$
\begin{aligned}
& \int_{\mathcal{D}} a_{M}^{-1}(\mathbf{x}, \mathbf{y}) \vec{u}(\mathbf{x}, \mathbf{y}) \cdot \vec{v}(\mathbf{x}) \mathrm{d} \mathbf{x}-\int_{\mathcal{D}} p(\mathbf{x}, \mathbf{y}) \nabla \cdot \vec{v}(\mathbf{x}) \mathrm{d} \mathbf{x}=-\int_{\partial \mathcal{D}_{\mathrm{D}}} g(\mathbf{x}) \vec{v}(\mathbf{x}) \cdot \vec{n} \mathrm{~d} s, \\
&-\int_{\mathcal{D}} q(\mathbf{x}) \nabla \cdot \vec{u}(\mathbf{x}, \mathbf{y}) \mathrm{d} \mathbf{x}=-\int_{\mathcal{D}} f(\mathbf{x}) q(\mathbf{x}) \mathrm{d} \mathbf{x}
\end{aligned}
$$

for all $q(\mathbf{x}) \in Q$ and $\vec{v}(\mathbf{x}) \in V$. This is a saddle point problem of the form (1.1) with

$$
a(\vec{u}(\cdot, \mathbf{y}), \vec{v}, \mathbf{y})=\int_{\mathcal{D}} a_{M}^{-1}(\mathbf{x}, \mathbf{y}) \vec{u}(\mathbf{x}, \mathbf{y}) \cdot \vec{v}(\mathbf{x}) \mathrm{d} \mathbf{x}, \quad b(\vec{u}(\cdot, \mathbf{y}), q)=-\int_{\mathcal{D}} q(\mathbf{x}) \nabla \cdot \vec{u}(\mathbf{x}, \mathbf{y}) \mathrm{d} \mathbf{x} .
$$

The above Darcy flow problem serves as a representative example from the class of parameterdependent saddle point problems (1.1) where the $\mathbf{y}$ dependence only appears in the bilinear form $a(\cdot, \cdot, \mathbf{y})$. Moreover, since the inverse of the diffusion coefficient appears, $a(\cdot, \cdot, \mathbf{y})$ has non-affine dependence on the parameters $\mathbf{y}$. For this problem, we note that $\nabla \cdot V \subset Q$ and so $V_{0}$ in (2.1) contains functions in $V$ which are divergence-free. The coercivity condition (2.2) is satisfied on $V_{0}$, with $\alpha(\mathbf{y})=a_{\min }(\mathbf{y})$. The inf-sup condition (2.3) is also satisfied, see [9]. To perform forward UQ for (3.7) using sampling methods such as SCMs (see Section 5), we will need to be able to approximate $\vec{u}(\cdot, \mathbf{y})$ and $p(\cdot, \mathbf{y})$, for many different choices of $\mathbf{y}$, cheaply and efficiently, and to estimate statistical quantities such as

$$
\mathbb{E}[\vec{u}(\mathbf{x}, \cdot)]=\int_{\Gamma} \rho(\mathbf{y}) \vec{u}(\mathbf{x}, \mathbf{y}) \mathrm{d} \mathbf{y}, \quad \mathbb{E}[p(\mathbf{x}, \cdot)]=\int_{\Gamma} \rho(\mathbf{y}) p(\mathbf{x}, \mathbf{y}) \mathrm{d} \mathbf{y},
$$

as well as higher order moments. Using appropriate mixed finite element methods (MFEMs) allows us to approximate both the velocity and pressure to the same order of accuracy for each $\mathbf{y}$ of interest and to ensure that mass is locally conserved on the elements, see [18, 23]. 
3.1. High fidelity problem. By introducing finite-dimensional subspaces $V_{h} \subset V, Q_{h} \subset Q$, the discrete analogue of (3.7) is a saddle point problem of the form (2.4). We use Raviart-Thomas mixed finite elements, see [9] for details, to ensure that (2.7) is satisfied. For these spaces, we have $\nabla \cdot V_{h} \subset Q_{h}$ so $V_{0, h}$ contains divergence-free functions and (2.5) is satisfied on $V_{0, h}$ with $\alpha_{h}(\mathbf{y})=a_{\min }(\mathbf{y})$. In Section 6, we use lowest-order Raviart-Thomas elements, which guarantees that, if realisations of the diffusion coefficient are smooth enough, the high-fidelity discretisation errors satisfy $\left\|\vec{u}(\cdot, \mathbf{y})-\vec{u}_{h}(\cdot, \mathbf{y})\right\|_{\mathrm{H}(\operatorname{div}, \mathcal{D})}=O(h)$ and $\left\|p(\cdot, \mathbf{y})-p_{h}(\cdot, \mathbf{y})\right\|_{L^{2}(\mathcal{D})}=O(h)$.

Next, we derive the linear systems associated with the high fidelity problem. Given a basis $\left\{\phi_{i}(\mathbf{x}), i=1, \ldots, N_{p}\right\}$ for $Q_{h}$ and a basis $\left\{\vec{\varphi}_{i}(\mathbf{x}), i=1, \ldots, N_{u}\right\}$ for $V_{h}$, where $N_{u}=\operatorname{dim} V_{h}$ and $N_{p}=\operatorname{dim} Q_{h}$, we define the matrices $A(\mathbf{y}) \in \mathbb{R}^{N_{u} \times N_{u}}$ and $B \in \mathbb{R}^{N_{p} \times N_{u}}$ with entries

$$
\begin{aligned}
{[A(\mathbf{y})]_{i j} } & =\int_{\mathcal{D}} a_{M}^{-1}(\mathbf{x}, \mathbf{y}) \vec{\varphi}_{i}(\mathbf{x}) \cdot \vec{\varphi}_{j}(\mathbf{x}) \mathrm{d} \mathbf{x}, & & i, j=1, \ldots, N_{u}, \\
B_{k j} & =-\int_{\mathcal{D}} \phi_{k}(\mathbf{x}) \nabla \cdot \vec{\varphi}_{j}(\mathbf{x}) \mathrm{d} \mathbf{x}, & & j=1, \ldots, N_{u}, k=1, \ldots, N_{p},
\end{aligned}
$$

and vectors $\mathbf{g} \in \mathbb{R}^{N_{u}}$ and $\mathbf{f} \in \mathbb{R}^{N_{p}}$ with entries

$$
g_{i}=-\int_{\partial \mathcal{D}_{\mathrm{D}}} g(\mathbf{x}) \vec{\varphi}_{i}(\mathbf{x}) \cdot \vec{n} \mathrm{~d} s, i=1, \ldots, N_{u}, \quad f_{j}=-\int_{\mathcal{D}} f(\mathbf{x}) \phi_{j}(\mathbf{x}) \mathrm{d} \mathbf{x}, j=1, \ldots, N_{p} .
$$

Then, for a fixed $\mathbf{y} \in \Gamma$, the parameter-dependent high fidelity problem can be written as

$$
\left[\begin{array}{cc}
A(\mathbf{y}) & B^{\top} \\
B & 0
\end{array}\right]\left[\begin{array}{l}
\mathbf{u}(\mathbf{y}) \\
\mathbf{p}(\mathbf{y})
\end{array}\right]=\left[\begin{array}{l}
\mathbf{g} \\
\mathbf{f}
\end{array}\right]
$$

where $\mathbf{u}(\mathbf{y})$ and $\mathbf{p}(\mathbf{y})$ are the coefficients that represent the snapshot pair $\left(\vec{u}_{h}(\cdot, \mathbf{y}), p_{h}(\cdot, \mathbf{y})\right)$. The coefficient matrix is of size $N_{h} \times N_{h}$, where $N_{h}=N_{u}+N_{p}$, and is sparse. Solution methods for indefinite systems of the form (3.10) are discussed in [6]. Iterative methods such as MINRES $[27,36]$, with preconditioning $[38,43]$, which exploit sparsity, can be implemented at $\mathcal{O}\left(N_{h}\right)$ cost.

To construct reduced spaces $V_{R}$ and $Q_{R}$ that satisfy (2.11) we need to compute $N_{R}$ supremizer functions $T q$, defined as in (2.16), for the pressure snapshots $q=p_{h}\left(\cdot, \mathbf{y}_{i}\right) \in Q_{h}, i=1, \ldots, N_{R}$. Given any $q \in Q_{h}$, we can expand $T q \in V_{h}$ as

$$
T q=\sum_{i=1}^{N_{u}} t_{i} \vec{\varphi}_{i}
$$

Recall that there is no $\mathbf{y}$ dependence in the bilinear form $b(\cdot, \cdot)$. Substituting (3.11) into (2.15) and setting $\vec{v}=\vec{\varphi}_{j}$ and $q=\sum_{i=1}^{N_{p}} q_{i} \phi_{i}$ yields

$$
\sum_{i=1}^{N_{u}} t_{i}\left\langle\vec{\varphi}_{i}(\mathbf{x}), \vec{\varphi}_{j}(\mathbf{x})\right\rangle_{V}=-\sum_{i=1}^{N_{p}}\left(\int_{\mathcal{D}} \nabla \cdot \vec{\varphi}_{j}(\mathbf{x}) \phi_{i}(\mathbf{x}) \mathrm{d} \mathbf{x}\right) q_{i}, \quad j=1, \ldots, N_{u} .
$$

Hence, the vector of coefficients $\mathbf{t}\left(\mathbf{y}_{i}\right)$ associated with the supremizer function $\operatorname{Tp}_{h}\left(\cdot, \mathbf{y}_{i}\right), i=$ $1, \ldots, N_{R}$, can be computed by solving the linear system

$$
(M+D) \mathbf{t}\left(\mathbf{y}_{i}\right)=B^{\top} \mathbf{q}
$$

where the matrices $M$ and $D$ are defined as

$$
M_{i j}=\int_{\mathcal{D}} \vec{\varphi}_{i}(\mathbf{x}) \cdot \vec{\varphi}_{j}(\mathbf{x}) \mathrm{d} \mathbf{x}, \quad D_{i j}=\int_{\mathcal{D}}\left(\nabla \cdot \vec{\varphi}_{i}(\mathbf{x})\right)\left(\nabla \cdot \vec{\varphi}_{j}(\mathbf{x})\right) \mathrm{d} \mathbf{x}, \quad i, j=1, \ldots, N_{u} .
$$


The cost of solving (3.13) for each $\mathbf{y} \in \Gamma$ can be $\mathcal{O}\left(N_{u}\right)$ if we use iterative methods, but care must be taken. The matrix $M+D$ is a discrete representation of the $\mathrm{H}(\operatorname{div}, \mathcal{D})$ norm since

$$
\|\vec{v}\|_{\mathrm{H}(\operatorname{div}, \mathcal{D})}^{2}=\mathbf{v}^{\top}(M+D) \mathbf{v}, \quad \vec{v} \in V
$$

It does not represent an elliptic operator. In the reduced formulation of parameter-dependent Stokes and steady-state Navier-Stokes problems, computing the supremizer functions involves solving elliptic (Poisson) problems, see [24, 41, 42]. Standard multigrid methods, for example, will work well in that case, but not for (3.13). It should also be noted that the matrix $M+D$ can also be used to construct an $h$-optimal block-diagonal preconditioner

$$
P=\left(\begin{array}{cc}
M+D & 0 \\
0 & N
\end{array}\right)
$$

for the high fidelity system (3.10) see [38], where $N$ is the pressure mass matrix, with entries

$$
N_{k s}=\int_{\mathcal{D}} \phi_{k}(\mathbf{x}) \cdot \phi_{s}(\mathbf{x}), \quad k, s=1, \ldots, N_{p} .
$$

3.2. Reduced problem. Given reduced spaces $V_{R} \subset V_{h}$ and $Q_{R} \subset Q_{h}$, we can derive a parameter-dependent reduced problem of the form $(2.8)$ with $a(\cdot, \cdot, \mathbf{y}): V_{R} \times V_{R} \rightarrow \mathbb{R}$ and $b(\cdot, \cdot): V_{R} \times Q_{R} \rightarrow \mathbb{R}$ defined as in (3.8). We construct $V_{R}$ and $Q_{R}$ as in (2.17) so that the inf-sup condition (2.11) is satisfied. However, it is not clear whether (2.9) is satisfied in the $\mathrm{H}(\operatorname{div}, \mathcal{D})$ norm, unless $\nabla \cdot V_{R} \subset Q_{R}$. Note, however, that we do have coercivity in the $L^{2}(D)$ norm since

$$
a\left(\vec{v}_{R}, \vec{v}_{R}, \mathbf{y}\right) \geq a_{\min }(\mathbf{y})\left\|\vec{v}_{R}\right\|_{L^{2}(D)}, \quad \forall \vec{v}_{R} \in V_{R}
$$

We assume that we have constructed two reduced basis matrices $Q_{u} \in \mathbb{R}^{N_{u} \times 2 N_{R}}$ and $Q_{p} \in$ $\mathbb{R}^{N_{p} \times N_{R}}$ that represent discrete versions of the reduced spaces $V_{R}$ and $Q_{R}$, respectively, and define

$$
Q=\left[\begin{array}{cc}
Q_{u} & 0 \\
0 & Q_{p}
\end{array}\right]
$$

We describe how to construct these in Section 4.1. Then, for each $\mathbf{y} \in \Gamma$, the linear system associated with the reduced Darcy flow problem is,

$$
Q^{\top}\left[\begin{array}{cc}
A(\mathbf{y}) & B^{\top} \\
B & 0
\end{array}\right] Q\left[\begin{array}{l}
\mathbf{u}_{R}(\mathbf{y}) \\
\mathbf{p}_{R}(\mathbf{y})
\end{array}\right]=Q^{\top}\left[\begin{array}{l}
\mathbf{g} \\
\mathbf{f}
\end{array}\right] .
$$

The coefficient matrix in (3.19) is of size $3 N_{R} \times 3 N_{R}$, but is dense rather than sparse as in (3.10). Using direct methods, the cost of solving (3.19) for each $\mathbf{y} \in \Gamma$ is $\mathcal{O}\left(N_{R}^{3}\right)$. Hence, when $N_{R} \ll N_{h}$ we expect that the cost of solving (3.19) will be significantly cheaper than solving (3.10). It may be, however, that while $N_{R} \ll N_{h}$ we have $N_{R}^{3}>N_{h}$, and so solving (3.19) may not actually be cheaper than solving (3.10). The size of the reduced basis $N_{R}$ is key, and this is of course, problem-dependent. In [20] the authors consider using preconditioned iterative methods for solving the dense reduced systems that arise from the reduced formulation of a linear elliptic PDE. For each $\mathbf{y} \in \Gamma$ the cost is $\mathcal{O}\left(k N_{R}^{2}\right)$, where $k$ is the number of iterations. This can be an effective strategy in some problems, as long as the number of iterations $k$ is small.

We can assemble the system (3.19) from the high fidelity matrices and vectors via

$$
A_{R}(\mathbf{y})=Q_{u}^{\top} A(\mathbf{y}) Q_{u}, \quad B_{R}=Q_{p}^{\top} B Q_{u}, \quad \mathbf{g}_{R}=Q_{u}^{\top} \mathbf{g}, \quad \mathbf{f}_{R}=Q_{p}^{\top} \mathbf{f} .
$$


However this will incur a cost that depends on $N_{h}$ and so we cannot construct (3.19) directly in the online stage, for each $\mathbf{y} \in \Gamma$ of interest. To construct (3.19) in a way that the cost is independent of $N_{h}$, we need to be able to decompose the matrix $A(\mathbf{y})$ as

$$
A(\mathbf{y})=\sum_{k=1}^{K} A_{k} \theta_{k}(\mathbf{y})
$$

This means we need an affine expansion of $a(\cdot, \cdot, \mathbf{y})$ as in (1.2). For the Darcy flow problem, we require that the inverse of the diffusion coefficient $a_{M}(\mathbf{x}, \mathbf{y})$ is of the form

$$
a_{M}^{-1}(\mathbf{x}, \mathbf{y})=\sum_{k=1}^{K} \theta_{k}(\mathbf{y}) a_{k}(\mathbf{x})
$$

If this is the case, we can define $K$ parameter independent matrices $A_{k} \in \mathbb{R}^{N_{u} \times N_{u}}$ with entries

$$
\left[A_{k}\right]_{i j}=\int_{\mathcal{D}} a_{k}(\mathbf{x}) \vec{\varphi}_{i}(\mathbf{x}) \cdot \vec{\varphi}_{j}(\mathbf{x}) \mathrm{d} \mathbf{x}, \quad i, j=1, \ldots, N_{u},
$$

for $k=1, \ldots, K$. Exploiting this we have

$$
A_{R}(\mathbf{y})=Q_{u}^{\top} A(\mathbf{y}) Q_{u}=Q_{u}^{\top} \sum_{k=1}^{K} A_{k} \theta_{k}(\mathbf{y}) Q_{u}=\sum_{k=1}^{K} \theta_{k}(\mathbf{y}) Q_{u}^{\top} A_{k} Q_{u} .
$$

Hence, we can precompute and store the matrices $\left\{Q_{u}^{\top} A_{k} Q_{u}\right\}_{k=1}^{K}$ offline, allowing for the system (3.19) to be assembled for the $\mathbf{y} \in \Gamma$ of interest online, at a cost independent of $N_{h}$.

For the Darcy flow problem, however, the coefficient $a_{M}(\mathbf{x}, \mathbf{y})$ appears as $a_{M}^{-1}(\mathbf{x}, \mathbf{y})$ in the bilinear form $a(\cdot, \cdot, \mathbf{y})$ and so we do not have a decomposition of the form (3.20). To resolve this, we apply a discrete empirical interpolation method (DEIM) to obtain an approximation $a_{M}^{-1}(\mathbf{x}, \mathbf{y}) \approx \sum_{k=1}^{K} \theta_{k}(\mathbf{y}) a_{k}(\mathbf{x})$. We describe how to do this in Section 4.2. This yields a matrix approximation $\tilde{A}(\mathbf{y}) \approx A(\mathbf{y})$ and we solve the RB-DEIM system

$$
Q^{\top}\left[\begin{array}{cc}
\tilde{A}(\mathbf{y}) & B^{\top} \\
B & 0
\end{array}\right] Q\left[\begin{array}{c}
\tilde{\mathbf{u}}_{R}(\mathbf{y}) \\
\tilde{\mathbf{p}}_{R}(\mathbf{y})
\end{array}\right]=Q^{\top}\left[\begin{array}{l}
\mathbf{g} \\
\mathbf{f}
\end{array}\right], \quad \tilde{A}(\mathbf{y}):=\sum_{k=1}^{K} A_{k} \theta_{k}(\mathbf{y}) .
$$

We use $\mathbf{u}_{R}$ and $\mathbf{p}_{R}$ to denote the solution to the RB system (3.19) and $\tilde{\mathbf{u}}_{R}$ and $\tilde{\mathbf{p}}_{R}$ to denote the solution to the RB-DEIM system (3.21).

To end this section, we summarise the key features of the parameter-dependent Darcy flow saddle point problem. First, the bilinear form $a(\cdot, \cdot, \mathbf{y})$ depends non-affinely on the vector of parameters $\mathbf{y}$. We deal with this by using DEIM, taking care to balance the DEIM error with the reduced basis error associated with $V_{R}$ and $Q_{R}$ (see Section 6). Second, the reduced problem does not automatically inherit coercivity from the high fidelity problem in the $\mathrm{H}(\operatorname{div}, \mathcal{D})$ norm, so the standard reduced basis error estimates do not immediately apply. Finally, computing the supremizer functions $T p_{h}\left(\cdot, \mathbf{y}_{i}\right)$ that are needed to construct a pair of inf-sup stable reduced spaces requires the solution of $N_{R}$ discrete $\mathrm{H}(\operatorname{div}, \mathcal{D})$ problems.

4. Reduced basis method offline. In this section we describe the offline procedure for constructing the reduced spaces $V_{R}$ and $Q_{R}$. In particular we describe how to construct the matrices $Q_{u}$ and $Q_{p}$ and a DEIM approximation to the inverse of the diffusion coefficient. 
4.1. Reduced basis construction. The reduced basis matrices $Q_{u}$ and $Q_{p}$ are given by

$$
Q_{u}:=\left[\mathbf{u}\left(\mathbf{y}_{1}\right), \ldots, \mathbf{u}\left(\mathbf{y}_{N_{R}}\right), \mathbf{t}\left(\mathbf{y}_{1}\right), \ldots, \mathbf{t}\left(\mathbf{y}_{N_{R}}\right)\right] \in \mathbb{R}^{N_{u} \times 2 N_{R}},
$$

and

$$
Q_{p}:=\left[\mathbf{p}\left(\mathbf{y}_{1}\right), \ldots, \mathbf{p}\left(\mathbf{y}_{N_{R}}\right)\right] \in \mathbb{R}^{N_{p} \times N_{R}},
$$

where $\left(\mathbf{u}\left(\mathbf{y}_{i}\right), \mathbf{p}\left(\mathbf{y}_{i}\right)\right)$ are the coefficients associated with the snapshot pair $\left(\vec{u}_{h}\left(\cdot, \mathbf{y}_{i}\right), p_{h}\left(\cdot, \mathbf{y}_{i}\right)\right)$ and $\mathbf{t}\left(\mathbf{y}_{i}\right)$ is the vector of coefficients representing the supremizer function $T p_{h}\left(\cdot, \mathbf{y}_{i}\right), i=1, \ldots, N_{R}$. To construct the matrices, we have to solve $N_{R}$ high fidelity mixed finite element problems and $N_{R}$ discrete $\mathrm{H}(\operatorname{div}, \mathcal{D})$ problems. In this paper, we select the points $\left\{\mathbf{y}_{i}\right\}_{i=1}^{N_{R}}$ using a multilevel approach which is based on the algorithm presented in [22] for scalar elliptic PDEs. In our experience, it generally gives the quickest offline times (see Section 6), compared to more standard methods like greedy algorithms [39, Chapter 7], [31, Section 3.2.2] and the proper orthogonal decomposition (POD) [39, Chapter 6], [31, Section 3.2.1].

First, we must choose a training set, a finite set of points $\Theta \subset \Gamma$. These points could be chosen randomly, or deterministically. For example, $\Theta$ could be a set of sparse grid points (see Section 5). The main requirement is that $\Theta$ should be rich enough so that the resulting reduced bases yield accurate enough approximations to the high fidelity problem for any $\mathbf{y} \in \Gamma$ that may be encountered online. If the user has a priori knowledge about the set of points to be encountered online, then this information can be used to design a suitable training set. In this work, we choose nested sets of points $\Theta_{l}$, indexed by a level number $l$, so that $\Theta_{l-1} \subset \Theta_{l}$.

Next, we must select an error estimator to assess the error between the high fidelity approximation and a reduced approximation for a given $\mathbf{y} \in \Gamma$. We use the error indicator

$$
\Delta_{R}(\mathbf{y})=\left\|\left[\begin{array}{l}
\mathbf{g} \\
\mathbf{f}
\end{array}\right]-\left[\begin{array}{cc}
A(\mathbf{y}) & B^{\top} \\
B & 0
\end{array}\right]\left[\begin{array}{l}
Q_{u} \mathbf{u}_{R}(\mathbf{y}) \\
Q_{p} \mathbf{p}_{R}(\mathbf{y})
\end{array}\right]\right\|_{2} /\left\|\left[\begin{array}{l}
\mathbf{g} \\
\mathbf{f}
\end{array}\right]\right\|_{2},
$$

(the discrete relative residual error). This is a simple to compute approximation that, unlike a posteriori error estimators for the reduced basis error $\left\|\vec{u}_{h}(\cdot, \mathbf{y})-\vec{u}_{R}(\cdot, \mathbf{y})\right\|_{V}+\left\|p_{h}(\cdot, \mathbf{y})-p_{R}(\cdot, \mathbf{y})\right\|_{Q}$, does not require the computation of coercivity or inf-sup constants. However, there is no guarantee that $\Delta_{R}(\mathbf{y})$ is an upper bound for the true error, so our RB method is not 'certified' in the usual sense. To control the accuracy, we choose a tolerance $\epsilon_{1}>0$ and select a point $\mathbf{y}$ to help construct the reduced bases only if $\Delta_{R}(\mathbf{y})>\epsilon_{1}$.

To initialise $Q_{u}$ and $Q_{p}$, we first solve the systems (3.10) and (3.13) for a single point $\mathbf{y}_{0}$, for example the zero vector. Then, for each level $l=1, \ldots, L$, we loop through each point $\mathbf{y}_{j} \in \Theta_{l} \backslash \Theta_{l-1}$, for $j=1, \ldots, M_{l}$, where $M_{l}=\left|\Theta_{l} \backslash \Theta_{l-1}\right|$, solve the reduced system (3.19), and compute $\Delta_{R}\left(\mathbf{y}_{j}\right)$. If $\Delta_{R}\left(\mathbf{y}_{j}\right)>\epsilon_{1}$ then we solve the high fidelity problem and update the current bases immediately with a new snapshot pair corresponding to the point $\mathbf{y}_{j}$, as well as a supremizer function. Otherwise, we accept that the current reduced approximation for $\mathbf{y}_{j}$ is good enough. The detailed procedure is presented in Algorithm 1. The matrices $Q_{u}$ and $Q_{p}$ are constructed one column at a time. For numerical stability, we orthonormalise the columns using a QR factorisation. Once the iteration has terminated, we obtain the matrices $Q_{p} \in \mathbb{R}^{N_{p} \times N_{R}}$ and $Q_{u} \in \mathbb{R}^{N_{u} \times 2 N_{R}}$. Note that Algorithm 1 differs from the standard greedy algorithm as we do not update our reduced bases with the $\mathbf{y} \in \Theta_{l}$ that maximizes $\Delta_{R}(\mathbf{y})$ at each step. This means we only need to solve (3.19) once for each $\mathbf{y} \in \Theta_{l}$. In Section 6 we present numerical experiments showing that Algorithm 1 is significantly cheaper than the standard greedy algorithm and achieves similar values of $N_{R}$ in the construction of the matrices $Q_{u}$ and $Q_{p}$.

The costs involved in Algorithm 1 are summarised in Table 4.1. Let $N_{R_{l}}^{j}$ denote the number of high fidelity systems that have been solved after $l$ outer iterations and $j$ inner iterations, so 


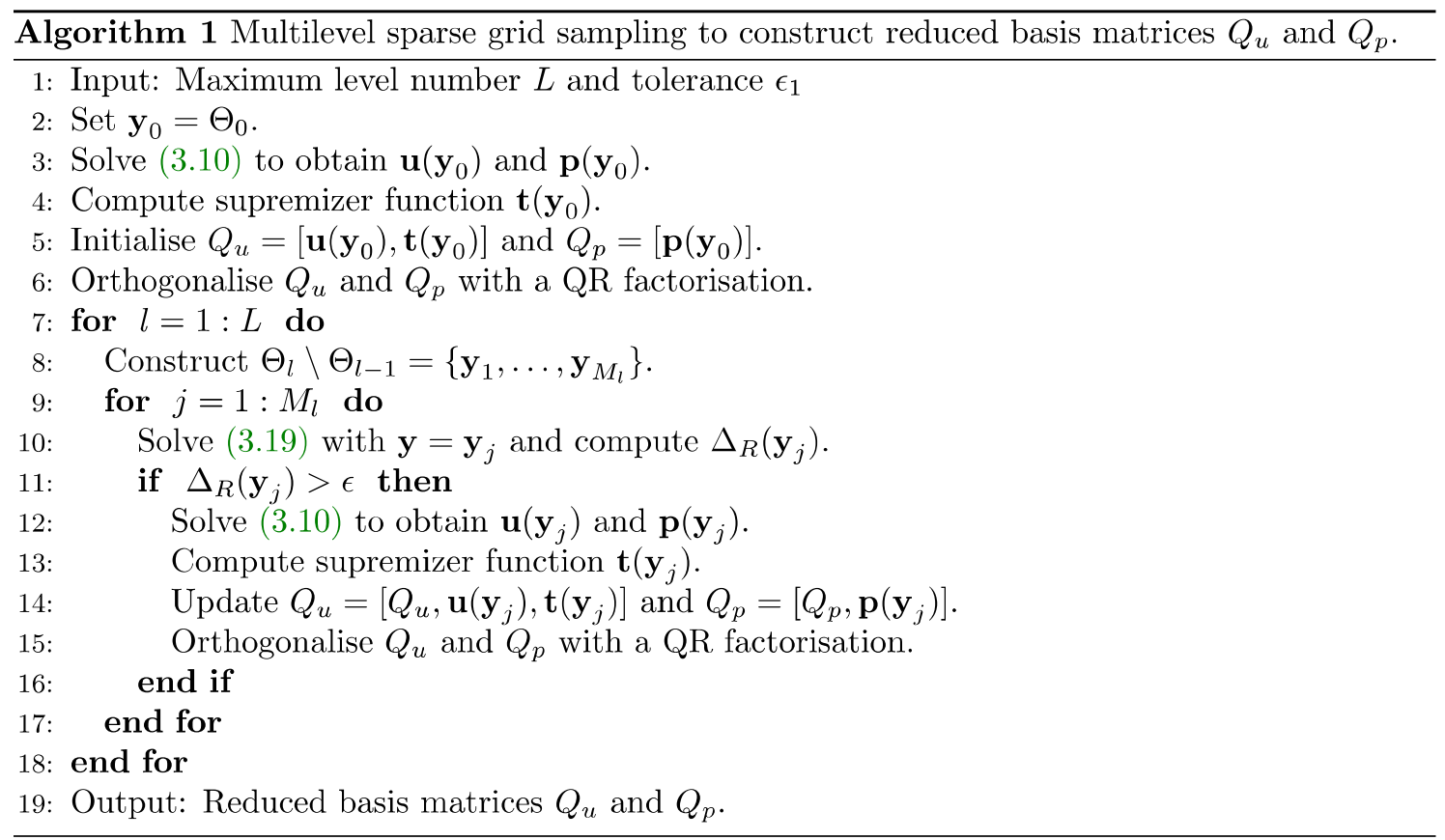

TABLE 4.1

Summary of costs associated with Algorithm 1.

\begin{tabular}{|c|c|}
\hline Task & Cost \\
\hline Assemble reduced system matrices $A_{R}(\mathbf{y})$ & $\sum_{l=1}^{L} \sum_{j=1}^{M_{l}} \mathcal{O}\left(N_{h}^{2} N_{R_{l-1}}^{j-1}+N_{h}\left(N_{R_{l-1}}^{j-1}\right)^{2}\right)$ \\
Solve reduced systems (3.19) & $\sum_{l=1}^{L} \sum_{j=1}^{M_{l}} \mathcal{O}\left(\left(N_{R_{l}}^{j}\right)^{3}\right)$ \\
Evaluate error indicator (4.3) & $\sum_{l=1}^{L} \sum_{j=1}^{M_{l}} \mathcal{O}\left(N_{h} N_{R_{l-1}}^{j-1}+N_{h}^{2}\right)$ \\
Solve (3.10) \& (3.13) & $N_{R} \times \mathcal{O}\left(N_{h}\right)$ \\
Orthonormalise columns of $Q_{u}$ and $Q_{p}$ & $\sum_{k=1}^{N_{R}} \mathcal{O}\left(k^{2} N_{h}\right)$ \\
\hline
\end{tabular}

that the sizes of the reduced bases at that stage are $2 N_{R_{l}}^{j}$ and $N_{R_{l}}^{j}$ for the velocity and pressure, respectively. At the $l^{\text {th }}$ outer iteration, and $j^{\text {th }}$ inner iteration, we have to assemble and solve (3.19) with the current reduced spaces, and evaluate (4.3) for $\mathbf{y}_{j} \in \Theta_{l} \backslash \Theta_{l-1}$. Over the course of the iteration, we also need to solve $N_{R}$ high fidelity and supremiser systems to augment the reduced bases, where $N_{R}=N_{R_{L}}^{M_{L}}$. Finally, the columns of $Q_{u}$ and $Q_{p}$ are orthonormalised in each iteration. The total cost therefore depends on $N_{h}^{2}$ and $N_{R}^{3}$.

4.2. DEIM. The discrete empirical interpolation method (DEIM) [12], see also the empirical interpolation method [3], ${ }^{1}$ allows us to generate an approximation to a parameter-dependent function $f(\mathbf{x}, \mathbf{y})$, of the form

$$
f(\mathbf{x}, \mathbf{y}) \approx \sum_{k=1}^{K} z_{k}(\mathbf{x}) \theta_{k}(\mathbf{y})
$$

\footnotetext{
${ }^{1}$ Contrary to what the names suggest, both of these methods are in fact 'discrete'.
} 


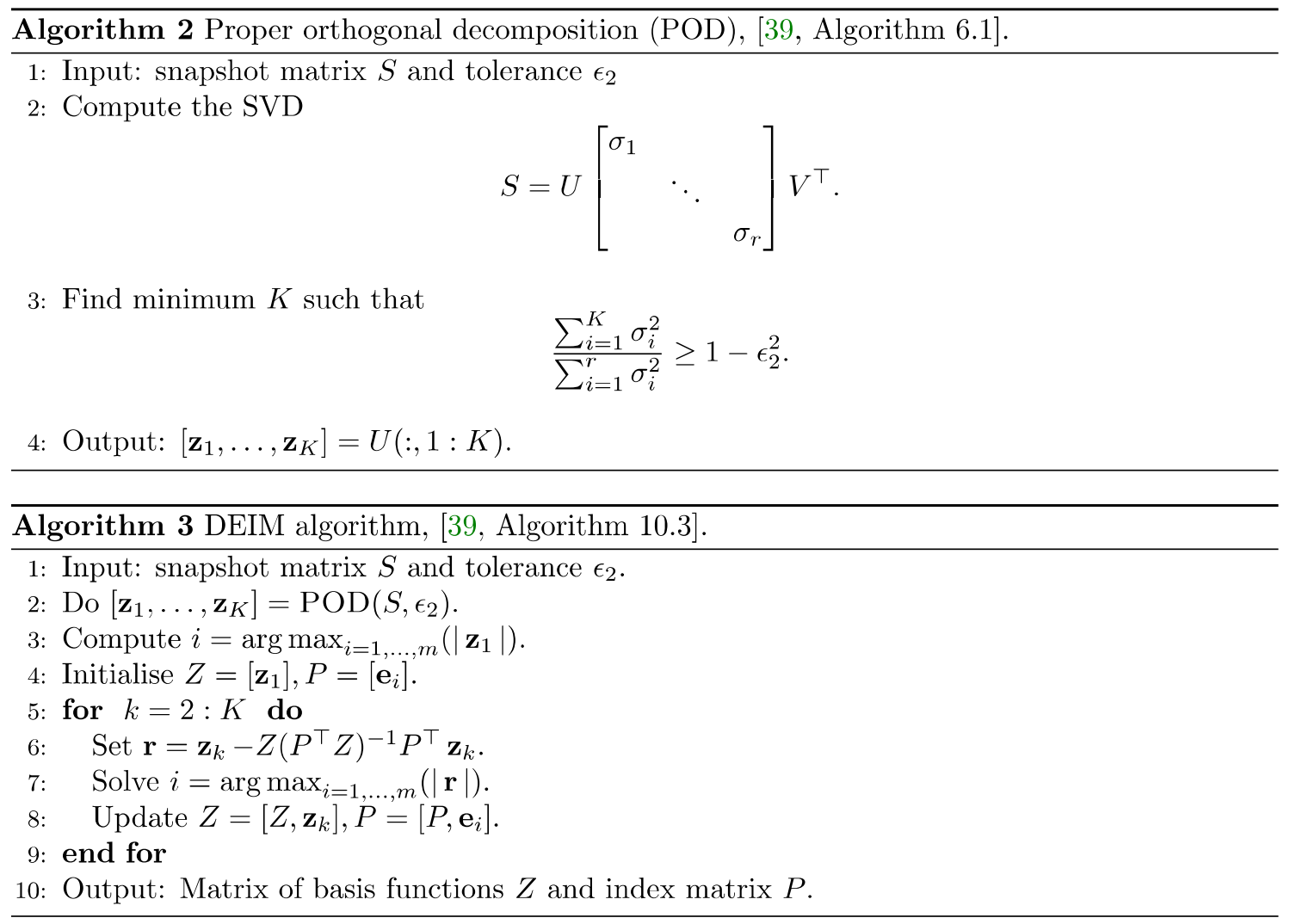

We employ the DEIM to approximate the coefficient $a_{M}^{-1}(\mathbf{x}, \mathbf{y})$ in the Darcy flow model. In the following description we summarise the procedure for a general parameter-dependent function $f(\mathbf{x}, \mathbf{y})$ to simplify notation. In practice, we generally have access to a vector $\mathbf{f}(\mathbf{y}) \in \mathbb{R}^{m}$, corresponding to the function $f(\mathbf{x}, \mathbf{y})$ evaluated at $m$ points $\mathbf{x}$ in the spatial domain $\mathcal{D}$ (for example, at points in a finite element mesh). The DEIM projects $\mathbf{f}(\mathbf{y})$ onto the subspace spanned by the $K$ columns of a chosen basis matrix $Z \in \mathbb{R}^{m \times K}$, with $K \ll m$.

Given a basis matrix $Z \in \mathbb{R}^{m \times K}$, the DEIM approximation to $\mathbf{f}(\mathbf{y}) \in \mathbb{R}^{m}$ can be written as

$$
\mathbf{f}_{K}(\mathbf{y})=Z \mathbf{c}(\mathbf{y}) \in \mathbb{R}^{m},
$$

where the $K$ columns of $Z$ represent the functions $z_{k}(\mathbf{x})$ in (4.4) evaluated at $m$ points $\mathbf{x} \in \mathcal{D}$, and the entries of $\mathbf{c}(\mathbf{y}) \in \mathbb{R}^{K}$ are the scalar coefficients $\theta_{k}(\mathbf{y})$ in (4.4) corresponding to a particular choice of $\mathbf{y}$. To construct $Z$ we must first select a set of points $\left\{\mathbf{y}_{i}\right\}_{i=1}^{n} \subset \Gamma$ to act as a training set. Given the training set, we then construct the snapshot matrix

$$
S=\left[\mathbf{f}\left(\mathbf{y}_{1}\right), \ldots, \mathbf{f}\left(\mathbf{y}_{n}\right)\right] \in \mathbb{R}^{m \times n}
$$

and compute its SVD, see [49], to obtain $S=U \Sigma V^{\top}$, where $\Sigma$ is the diagonal matrix of singular values in descending order and $U$ and $V$ are orthogonal matrices. The matrix $Z$ is chosen to be the first $K$ columns of $U$, where $K$ is the smallest number such that

$$
\frac{\sum_{i=1}^{K} \sigma_{i}^{2}}{\sum_{i=1}^{r} \sigma_{i}^{2}} \geq 1-\epsilon_{2}^{2}
$$

where $\epsilon_{2}$ is a chosen tolerance, and $r$ is the rank of $S$. This procedure is summarized in Algorithm 2. 
Once we have a basis matrix $Z$ we could, for a particular $\mathbf{y}$, determine the vector $\mathbf{c}(\mathbf{y})$ by solving $\mathbf{f}(\mathbf{y})=Z \mathbf{c}(\mathbf{y})$. However, this is an overdetermined system, so we instead demand that the equations are satisfied for only $K$ points $\mathbf{x} \in D$. The procedure for choosing these is called empirical interpolation and details are given in Algorithm 3. Given a set of $K$ indices, the algorithm constructs a matrix $P \in \mathbb{R}^{m \times K}$ whose columns are the columns of the $m \times m$ identity matrix associated with the chosen indices. Note that applying $P^{\top}$ to a vector $\mathbf{v} \in \mathbb{R}^{m}$ simply extracts $K$ components of v. $P$ does not need to be constructed. Given $Z$ and $P$ (or equivalently, the set of $K$ indices), for each $\mathbf{y}$ of interest, $\mathbf{c}(\mathbf{y})$ is found by solving the projected dense system

$$
\left(P^{\top} Z\right) \mathbf{c}(\mathbf{y})=P^{\top} \mathbf{f}(\mathbf{y}) .
$$

We can then write the final DEIM approximation as

$$
\mathbf{f}_{K}(\mathbf{y})=Z \mathbf{c}(\mathbf{y})=Z\left(P^{\top} Z\right)^{-1} P^{\top} \mathbf{f}(\mathbf{y}) .
$$

The main cost associated with Algorithms 2 and 3 is the SVD, though this is only done once offline. Given $n$ training points $\mathbf{y} \in \Gamma$ and evaluating $f(\mathbf{x}, \mathbf{y})$ at $m$ points $\mathbf{x} \in D$, the cost is $\mathcal{O}\left(m n^{2}\right)$. The number of points $n$ in the training set should be large enough to incorporate potential variability in the system. Once the DEIM iteration has terminated, we precompute and store the parameter independent matrices and vectors

$$
\left\{Q_{u}^{\top} A_{k} Q_{u}\right\}_{k=1}^{K}, \quad Q_{p}^{\top} B Q_{u}, \quad Q_{p}^{\top} \mathbf{g}, \quad Q_{u} \mathbf{f} .
$$

The cost of this assembly is $K \times \mathcal{O}\left(N_{h}^{2} N_{R}+N_{h} N_{R}^{2}\right)$. Online, for any given $\mathbf{y} \in \Gamma$ of interest, we additionally need to solve the dense $K \times K$ linear system (4.6) to obtain the coefficients $\mathbf{c}(\mathbf{y})$ needed to compute the matrix $\tilde{A}(\mathbf{y})$ in $(3.21)$. The cost of this is $\mathcal{O}\left(K^{3}\right)$. Therefore, as long as $K$ does not depend on $N_{h}$, the DEIM can be used online. If we also wish to evaluate the error indicator (4.3) online, then in order to make the cost independent of $N_{h}$, additional parameter independent matrices and vectors will need to be computed offline.

When we combine Algorithms 1 and 3 there are two error tolerances, $\epsilon_{1}$ and $\epsilon_{2}$. We find that choosing $\epsilon_{1}=\epsilon_{2}$ provides a less accurate RB-DEIM approximation when compared to the RB approximation without DEIM. Note that the SVD is performed on the full snapshot matrix $S$, so the offline cost is not actually affected by the choice of $\epsilon_{2}$. To balance the contributions of the reduced basis and DEIM errors our recommendation is that $\epsilon_{2}<\epsilon_{1}$ (see Section 6).

Remark 4.1. When $a_{M}^{-1}(\mathbf{x}, \mathbf{y})$ takes values close to zero, realisations of the DEIM approximation may be negative, making (3.21) ill-posed. We do not encounter this issue in the numerical experiments presented in Section 6. However, it may arise when, for example, the standard deviation is large. In that case, the DEIM approximation should be improved by increasing $K$.

The conventional wisdom is that large offline costs are to be expected and that the computational savings made online are worth the price paid offline. The offline costs are often not reported. Our view is that this is problem dependent. In an online experiment where real time information is required for many choices of $\mathbf{y} \in \Gamma$, the offline cost will not necessarily be important. However, if the main task is, say, to compute moments of the solution, by combining a RB method with a sampling method such as a stochastic collocation method (SCM), then the offline/online splitting is artificial. The user knows a priori for which choices of parameters the high-fidelity problem needs to be solved. In such scenarios, the cost of constructing the reduced bases must be optimized.

5. Reduced basis collocation. In this section we outline sparse grid stochastic collocation methods for forward UQ. To begin, we describe how to construct sparse grids on the parameter domain $\Gamma$ from combinations of sets of interpolation points in one dimension. The idea was first introduced by Smolyak in 1963 [46], but we follow [4]. Assuming that the $M$ underlying random 
variables in the problem are independent, we have a parameter domain of the form $\Gamma=\prod_{i=1}^{M} \Gamma_{i}$, where $\Gamma_{i} \subset \mathbb{R}$. Now, for $i=1, \ldots, M$, let $\Theta_{i}^{1}=\left\{y_{i}^{1}, \ldots, y_{i}^{n_{i}+1}\right\} \subset \Gamma_{i}$ denote a one-dimensional set of $n_{i}+1$ interpolation points such that

$$
n_{i}= \begin{cases}0, & i=1 \\ 2^{i-1}, & i>1\end{cases}
$$

Given an approximation level $l$, the sparse grid on $\Gamma$ is the set of points

$$
\Theta_{l}:=H(l, M)=\bigcup_{l \leq\|\mathbf{i}\|_{1}<l+M} \Theta_{i_{1}}^{1} \times \cdots \times \Theta_{i_{M}}^{1}
$$

where $\mathbf{i}=\left(i_{1}, \ldots, i_{M}\right)^{\top} \in \mathbb{N}^{M}$ is a multi-index. If the one-dimensional points are nested, in the sense that $\Theta_{i}^{1} \subset \Theta_{i+1}^{1}$, then the formula simplifies to

$$
\Theta_{l}:=H(l, M)=\bigcup_{\|\mathbf{i}\|_{1}=l+M-1} \Theta_{i_{1}}^{1} \times \cdots \times \Theta_{i_{M}}^{1} .
$$

Now, given a level number $l$ and the corresponding sparse grid $\Theta_{l}=\left\{\mathbf{y}_{1}, \ldots, \mathbf{y}_{N_{l}}\right\}$, where $N_{l}=\left|\Theta_{l}\right|$, the high fidelity stochastic collocation mixed FEM (SCMFEM) approximation to the solution $\vec{u}: D \times \Gamma \rightarrow \mathbb{R}$ and $p: D \times \Gamma \rightarrow \mathbb{R}$ of $(3.1)$, where $a^{-1}(\mathbf{x}, \omega)$ is replaced with $a_{M}^{-1}(\mathbf{x}, \mathbf{y})$ and $\mathbf{y}=\boldsymbol{\xi}(\omega)$, is given by

$$
\vec{u}_{h, \Theta}(\mathbf{x}, \mathbf{y})=\sum_{i=1}^{N_{l}} \vec{u}_{h}\left(\mathbf{x}, \mathbf{y}_{i}\right) L_{i}(\mathbf{y}), \quad p_{h, \Theta}(\mathbf{x}, \mathbf{y})=\sum_{i=1}^{N_{l}} p_{h}\left(\mathbf{x}, \mathbf{y}_{i}\right) L_{i}(\mathbf{y}),
$$

where $L_{i}: \Gamma \rightarrow \mathbb{R}$ is the $M$-dimensional Lagrange polynomial satisfying $L_{i}\left(\mathbf{y}_{j}\right)=\delta_{i j}$, for $j=$ $1, \ldots, N_{l}$. We can use (5.3) as a surrogate in any experiment where it is desired to approximate the solution to (3.5) at additional points $\mathbf{y} \in \Gamma$ that are not collocation points. Computing the high fidelity SCMFEM approximation requires the solution of (3.10) for all $\mathbf{y} \in \Theta_{l}$. The total cost is $N_{l} \times \mathcal{O}\left(N_{h}\right)$, which is infeasible in complex applications. Approximating statistical moments, such as (3.9), can be done on-the-fly, but if we wish to use (5.3) as a surrogate then we will need to store $N_{l}$ vectors of length $N_{h}$ for future use.

Reduced basis methods can be used in conjunction with stochastic collocation methods, such as in $[15,22]$ for scalar elliptic PDEs. If we replace the high fidelity approximations in (5.3) with RB-DEIM approximations then the new RB-DEIM-SCMFEM approximations are given by

$$
\vec{u}_{R, \Theta}(\mathbf{x}, \mathbf{y})=\sum_{i=1}^{N_{l}} \vec{u}_{R}\left(\mathbf{x}, \mathbf{y}_{i}\right) L_{i}(\mathbf{y}), \quad p_{R, \Theta}(\mathbf{x}, \mathbf{y})=\sum_{i=1}^{N_{l}} p_{R}\left(\mathbf{x}, \mathbf{y}_{i}\right) L_{i}(\mathbf{y}) .
$$

Using Algorithms 1 and 3, we can compute (5.4) by solving (3.21) for each $\mathbf{y} \in \Theta_{l}$. In Algorithm 1, we use $\Theta_{l}$ as the training set and the cost is $N_{l} \times \mathcal{O}\left(N_{R}^{3}+K^{3}\right)$. As long as $N_{R} \ll N_{h}$ and $K \ll N_{h}$ it will be cheaper to compute (5.4) than (5.3). Again, (3.9) can be approximated on-the-fly, but if we wish to make further use of (5.4) then we need only store $N_{l}$ vectors of length $3 N_{R}$.

In the framework of stochastic collocation we can think of the construction of either (5.3) or (5.4) as an offline computation, while the evaluation of either (5.3) or (5.4) for parameters $\mathbf{y} \in \Gamma$ that are not collocation points, or some other quantity of interest, as an online computation. As long as $N_{R} \ll N_{h}$ and $N_{R} \ll N_{l}$ we expect that reduced basis collocation (with DEIM approximation if necessary) will outperform high fidelity collocation. 
6. Numerical results. In this section we present some numerical results to illustrate the efficiency of using the RB-DEIM-SCMFEM scheme for the Darcy flow problem (3.1) with random coefficients. All computations are carried out using MATLAB on an Intel Xeon CPU with 2.50GHz. All linear systems, including (3.13), are solved using the MATLAB backslash function.

6.1. Non-affine test problems. Let $\mathcal{D}$ be a square domain and set $f(\mathbf{x})=0$. We consider (3.1) with $a(\mathbf{x}, \omega)=\exp (z(\mathbf{x}, \omega))$, where $z(\mathbf{x}, \omega)$ is a random field whose approximation, as a function of $M$ random variables, is $z_{M}(\mathbf{x}, \boldsymbol{\xi}(\omega))$, where $\boldsymbol{\xi}(\omega)=\left[\xi_{1}(\omega), \ldots, \xi_{M}(\omega)\right]^{\top}$. Choosing independent random variables $\xi_{k} \sim U(-\sqrt{3}, \sqrt{3})$ leads to the parameter domain $\Gamma=[-\sqrt{3}, \sqrt{3}]^{M}$. Now, working in terms of $\mathbf{y} \in \Gamma$, we assume that

$$
z_{M}(\mathbf{x}, \mathbf{y})=\sum_{k=1}^{M} \sqrt{\lambda_{k}} \phi_{k}(\mathbf{x}) y_{k},
$$

which has the structure of a truncated Karhunen-Loève expansion (3.3) with mean $\mu(\mathbf{x})=0$. We consider three examples. In the first two cases, $\lambda_{k} \rightarrow 0$ algebraically as $k \rightarrow \infty$, and in the third case, $\lambda_{k} \rightarrow 0$ exponentially as $k \rightarrow \infty$.

Example 6.1. Let $\mathcal{D}=[-1,1]^{2}$, set $p=1$ on $\{-1\} \times[-1,1], p=0$ on $\{1\} \times[-1,1]$, and choose homogeneous Neumann conditions for the velocity on $(-1,1) \times\{-1,1\}$. Here, we choose $\lambda_{k}$ and $\phi_{k}(\mathbf{x})$ in (6.1) to be eigenvalues and eigenfunctions, respectively, of the covariance function

$$
C\left(\mathbf{x}_{1}, \mathbf{x}_{2}\right)=\sigma^{2} \exp \left(\frac{-\left\|\mathbf{x}_{1}-\mathbf{x}_{2}\right\|_{1}}{c}\right), \quad \mathbf{x}_{1}, \mathbf{x}_{2} \in \mathcal{D}
$$

where $c$ is the correlation length, which we fix to be $c=2$, and $\sigma$ is the standard deviation of the random field $z(\mathbf{x}, \omega)$. These eigenpairs can be explicitly computed, see [33, Example 7.55].

Example 6.2. Let $\mathcal{D}=[0,1]^{2}$, set $p=1$ on $\{0\} \times[0,1], p=0$ on $\{1\} \times[0,1]$, and choose homogeneous Neumann conditions for the velocity on $(0,1) \times\{0,1\}$. This time, following an example in [19], we choose $\sqrt{\lambda_{k}}=\bar{\alpha} k^{-\tilde{\sigma}}$, and

$$
\phi_{k}(\mathbf{x}):=\cos \left(2 \pi \beta_{1}(k) x_{1}\right) \cos \left(2 \pi \beta_{2}(k) x_{2}\right), \quad \mathbf{x}=\left(x_{1}, x_{2}\right)^{T} \in \mathcal{D},
$$

where $k \in \mathbb{N}, \beta_{1}(k)=(k-l(k))(l(k+1)) / 2, \beta_{2}(k)=l(k)-\beta_{1}(k), l(k)=\lfloor-1 / 2+\sqrt{1 / 4+2 k}\rfloor$. The parameter $\tilde{\sigma}$ controls the rate of decay of the terms $\sqrt{\lambda_{k}}$. We consider two cases: $\tilde{\sigma}=2$ with $\bar{\alpha}=0.547$, and $\tilde{\sigma}=4$ with $\bar{\alpha}=0.832$.

Example 6.3. We choose the spatial domain $D$ and the boundary conditions as in Example 6.2. In addition, following [33, Example 9.37], we choose $\lambda_{0}=1 / 4, \phi_{0}(\mathbf{x})=1$, and

$$
\lambda_{i j}:=\frac{1}{4} \exp \left(-\pi\left(i^{2}+j^{2}\right)\right), \quad \phi_{i j}(\mathbf{x}):=2 \cos \left(i \pi x_{1}\right) \cos \left(j \pi x_{2}\right), \quad \mathbf{x}=\left(x_{1}, x_{2}\right)^{T} \in \mathcal{D},
$$

for $i, j \geq 1$. The indices $i$ and $j$ are associated with two one-dimensional problems. The values $\lambda_{i j}$, and corresponding functions $\phi_{i j}$, are then ordered in terms of a single index $k$ as in (6.1).

In each case, choosing $a_{M}(\mathbf{x}, \mathbf{y})=\exp \left(z_{M}(\mathbf{x}, \mathbf{y})\right)$ yields a problem of the form (3.5). For the spatial discretisation we use lowest-order square Raviart-Thomas elements associated with two meshes: the first gives $N_{p}=4,096$ and $N_{u}=8,192$ and the second gives $N_{p}=65,536$ and $N_{u}=131,072$. We construct the reduced basis matrices $Q_{u}$ and $Q_{p}$ using Algorithm 1 where the training set $\Theta_{l}$ is chosen as a set of Clenshaw-Curtis sparse grid points. Unless stated otherwise, we set $l=4$. Since $a_{M}^{-1}(\mathbf{x}, \mathbf{y})$ does not depend affinely on $\mathbf{y}$ we also construct a DEIM approximation using Algorithm 3 with tolerance $\epsilon_{2}=\epsilon_{1} / 10$ and a training set of 2000 randomly selected points $\mathbf{y} \in \Gamma$. We use the DEIM approximation to compute the matrices and vectors in (4.7) offline. This allows us to assemble and evaluate (3.21) online, at a cost that is independent of $N_{h}=N_{u}+N_{p}$. 
TABLE 6.1

Values of $N_{R}$ and $K$ required for Example 6.1 on two spatial meshes, as we vary the tolerance $\epsilon_{1}$, with fixed training set $\Theta_{4}$, and maximum relative residual errors $\delta_{1}$ and $\delta_{2}$ over 100 points $\mathbf{y} \in \Gamma$.

\begin{tabular}{|c|ccc|cc|}
\hline$M$ & $\epsilon_{1}$ & $N_{R}$ & $K$ & $\delta_{1}$ & $\delta_{2}$ \\
\hline \multirow{5}{*}{8} & $1 \mathrm{e}-3$ & 10 & 38 & $6.71 \mathrm{e}-4$ & $6.70 \mathrm{e}-4$ \\
& $1 \mathrm{e}-4$ & 26 & 60 & $6.15 \mathrm{e}-5$ & $6.20 \mathrm{e}-5$ \\
& $1 \mathrm{e}-5$ & 44 & 84 & $7.44 \mathrm{e}-6$ & $7.48 \mathrm{e}-6$ \\
& $1 \mathrm{e}-6$ & 67 & 114 & $1.01 \mathrm{e}-6$ & $1.01 \mathrm{e}-6$ \\
\hline \multirow{5}{*}{12} & $1 \mathrm{e}-3$ & 15 & 55 & $6.08 \mathrm{e}-4$ & $6.14 \mathrm{e}-4$ \\
& $1 \mathrm{e}-4$ & 37 & 87 & $6.79 \mathrm{e}-5$ & $6.80 \mathrm{e}-5$ \\
& $1 \mathrm{e}-5$ & 59 & 124 & $8.80 \mathrm{e}-6$ & $8.98 \mathrm{e}-6$ \\
& $1 \mathrm{e}-6$ & 96 & 166 & $8.52 \mathrm{e}-7$ & $8.63 \mathrm{e}-7$ \\
\hline
\end{tabular}

(a) $N_{h}=12,288, N_{p}=4096, N_{u}=8192$.

\begin{tabular}{|c|ccc|cc|}
\hline$M$ & $\epsilon_{1}$ & $N_{R}$ & $K$ & $\delta_{1}$ & $\delta_{2}$ \\
\hline \multirow{5}{*}{8} & $1 \mathrm{e}-3$ & 10 & 38 & $5.25 \mathrm{e}-4$ & $5.17 \mathrm{e}-4$ \\
& $1 \mathrm{e}-4$ & 26 & 60 & $6.97 \mathrm{e}-5$ & $6.99 \mathrm{e}-5$ \\
& $1 \mathrm{e}-5$ & 37 & 84 & $5.83 \mathrm{e}-6$ & $5.82 \mathrm{e}-6$ \\
& $1 \mathrm{e}-6$ & 59 & 114 & $8.65 \mathrm{e}-7$ & $8.66 \mathrm{e}-7$ \\
\hline \multirow{5}{*}{12} & $1 \mathrm{e}-3$ & 14 & 55 & $4.89 \mathrm{e}-4$ & $4.89 \mathrm{e}-4$ \\
& $1 \mathrm{e}-4$ & 30 & 88 & $8.13 \mathrm{e}-5$ & $8.12 \mathrm{e}-5$ \\
& $1 \mathrm{e}-5$ & 53 & 124 & $6.56 \mathrm{e}-6$ & $6.61 \mathrm{e}-6$ \\
& $1 \mathrm{e}-6$ & 85 & 168 & $9.19 \mathrm{e}-7$ & $9.23 \mathrm{e}-7$ \\
\hline
\end{tabular}

(b) $N_{h}=196,608, N_{p}=65,536, N_{u}=131,072$.

Comparison with high fidelity approximation. First, we consider Example 6.1. We fix $\sigma=0.25$ in (6.2) and choose two values for the number of terms $M$ in (6.1): $M=8$ and $M=12$, which corresponds to retaining $87 \%$ and $89 \%$ of the integral of the variance of the underlying random field $z(\mathbf{x}, \omega)$, respectively. After constructing the reduced bases and DEIM approximation, we look at the relative residual error in the high fidelity linear system, and compare the accuracy of solutions to the RB-DEIM model (3.21) and the RB model (3.19). Specifically, we compute

$$
\begin{aligned}
& \delta_{1}=\max _{\mathbf{y} \in \Theta}\left(\left\|\left[\begin{array}{l}
\mathbf{g} \\
\mathbf{f}
\end{array}\right]-\left[\begin{array}{cc}
A(\mathbf{y}) & B^{T} \\
B & 0
\end{array}\right]\left[\begin{array}{l}
Q_{u} \mathbf{u}_{R}(\mathbf{y}) \\
Q_{p} \mathbf{p}_{R}(\mathbf{y})
\end{array}\right]\right\|_{2} /\left\|\left[\begin{array}{l}
\mathbf{g} \\
\mathbf{f}
\end{array}\right]\right\|_{2}\right), \\
& \delta_{2}=\max _{\mathbf{y} \in \Theta}\left(\left\|\left[\begin{array}{l}
\mathbf{g} \\
\mathbf{f}
\end{array}\right]-\left[\begin{array}{cc}
A(\mathbf{y}) & B^{T} \\
B & 0
\end{array}\right]\left[\begin{array}{l}
Q_{u} \tilde{\mathbf{u}}_{R}(\mathbf{y}) \\
Q_{p} \tilde{\mathbf{p}}_{R}(\mathbf{y})
\end{array}\right]\right\|_{2} /\left\|\left[\begin{array}{l}
\mathbf{g} \\
\mathbf{f}
\end{array}\right]\right\|_{2}\right),
\end{aligned}
$$

as we decrease $\epsilon_{1}$, where $\Theta \subset \Gamma$ is a set of 100 randomly selected vectors of parameters. Results are presented in Table 6.1. We observe that the accuracies of the RB and the RB-DEIM approximations are similar in all cases, so that the reduced basis and DEIM contributions to the error are balanced. Hence $\epsilon_{2}=\epsilon_{1} / 10$ is a good choice for this problem. Furthermore we observe that both $N_{R}$, the required dimension of $Q_{R}$, and $K$, the required number of terms in the DEIM approximation, are effectively independent of $N_{h}$ but do increase as we decrease the tolerance $\epsilon_{1}$ and increase the number of parameters $M$.

Next, we look at the time taken to assemble and solve the high fidelity systems (3.10), the reduced systems (3.19) associated with non-affine coefficients, and the reduced systems (3.21) which incorporate DEIM approximation, denoted $T, t$ and $\tau$ respectively, while decreasing the tolerance $\epsilon_{1}$. Once the reduced bases have been constructed using the training set $\Theta_{4}$, we compute the average time taken to assemble and solve (3.10), (3.19), and (3.21), over an additional 100 points $\mathbf{y} \in \Gamma$. The results are presented in Table 6.2. We observe that the average high fidelity time $T$ depends on $N_{h}$ but does not, of course, depend on $\epsilon_{1}$. The average RB time $t$ depends on both $N_{h}$ and $\epsilon_{1}$ since we have to assemble $A_{R}(\mathbf{y})$ directly. The average RB-DEIM time $\tau$ does not depend on $N_{h}$ but does depend on $\epsilon_{1}$. In all cases the average cost of solving the reduced system with integrated DEIM approximation is the cheapest option. When $N_{h}=196,608$, the largest value of $N_{R}$ is 85 and so $N_{R} \ll N_{h}$. Of course, increasing $M$ and decreasing $\epsilon_{1}$ further will cause $\tau$ to increase and at some stage, $\tau$ will become larger than $T$.

Now, we compare the time taken to estimate $\mathbb{E}[\vec{u}]$ and $\mathbb{E}[p]$ using the RB-DEIM-SCMFEM approximation (5.4) and the high fidelity SCMFEM approximation (5.3) as we increase the number of collocation points $N_{l}$. The offline stage of the RB-DEIM-SCMFEM scheme involves constructing 
TABLE 6.2

Average time in seconds to solve the high fidelity systems (3.10) (T), RB systems (3.19) ( $t$ ) and RB-DEIM systems (3.21) $(\tau)$, over 100 points $\mathbf{y} \in \Gamma$ for Example 6.1 on two spatial meshes with training set $\Theta_{4}$.

\begin{tabular}{|c|ccc|ccc|}
\hline$M$ & $\epsilon_{1}$ & $N_{R}$ & $K$ & $T$ & $t$ & $\tau$ \\
\hline \multirow{5}{*}{8} & $1 \mathrm{e}-3$ & 10 & 38 & $5.01 \mathrm{e}-2$ & $1.65 \mathrm{e}-2$ & $2.30 \mathrm{e}-4$ \\
& $1 \mathrm{e}-4$ & 26 & 60 & $5.02 \mathrm{e}-2$ & $2.19 \mathrm{e}-2$ & $6.62 \mathrm{e}-4$ \\
& $1 \mathrm{e}-5$ & 44 & 84 & $5.01 \mathrm{e}-2$ & $2.67 \mathrm{e}-2$ & $1.43 \mathrm{e}-3$ \\
& $1 \mathrm{e}-6$ & 67 & 114 & $5.01 \mathrm{e}-2$ & $3.95 \mathrm{e}-2$ & $5.04 \mathrm{e}-3$ \\
\hline \multirow{5}{*}{12} & $1 \mathrm{e}-3$ & 15 & 55 & $5.00 \mathrm{e}-2$ & $1.84 \mathrm{e}-2$ & $3.41 \mathrm{e}-4$ \\
& $1 \mathrm{e}-4$ & 37 & 87 & $5.03 \mathrm{e}-2$ & $2.55 \mathrm{e}-2$ & $1.93 \mathrm{e}-3$ \\
& $1 \mathrm{e}-5$ & 59 & 124 & $5.00 \mathrm{e}-2$ & $3.36 \mathrm{e}-2$ & $3.97 \mathrm{e}-3$ \\
& $1 \mathrm{e}-6$ & 96 & 166 & $5.00 \mathrm{e}-2$ & $7.28 \mathrm{e}-2$ & $8.34 \mathrm{e}-3$ \\
\hline
\end{tabular}

(a) $N_{h}=12,288, N_{p}=4096, N_{u}=8192$.

\begin{tabular}{|c|ccc|ccc|}
\hline$M$ & $\epsilon_{1}$ & $N_{R}$ & $K$ & $T$ & $t$ & $\tau$ \\
\hline \multirow{5}{*}{8} & $1 \mathrm{e}-3$ & 10 & 38 & $1.23 \mathrm{e} 0$ & $2.91 \mathrm{e}-1$ & $7.74 \mathrm{e}-4$ \\
& $1 \mathrm{e}-4$ & 26 & 60 & $1.23 \mathrm{e} 0$ & $5.30 \mathrm{e}-1$ & $1.10 \mathrm{e}-3$ \\
& $1 \mathrm{e}-5$ & 37 & 84 & $1.22 \mathrm{e} 0$ & $8.11 \mathrm{e}-1$ & $1.68 \mathrm{e}-3$ \\
& $1 \mathrm{e}-6$ & 59 & 114 & $1.23 \mathrm{e} 0$ & $1.18 \mathrm{e} 0$ & $4.10 \mathrm{e}-3$ \\
\hline \multirow{4}{*}{12} & $1 \mathrm{e}-3$ & 14 & 55 & $1.23 \mathrm{e} 0$ & $3.63 \mathrm{e}-1$ & $9.71 \mathrm{e}-4$ \\
& $1 \mathrm{e}-4$ & 30 & 88 & $1.21 \mathrm{e} 0$ & $6.35 \mathrm{e}-1$ & $1.62 \mathrm{e}-3$ \\
& $1 \mathrm{e}-5$ & 53 & 124 & $1.22 \mathrm{e} 0$ & $1.07 \mathrm{e} 0$ & $3.59 \mathrm{e}-3$ \\
& $1 \mathrm{e}-6$ & 85 & 168 & $1.23 \mathrm{e} 0$ & $1.63 \mathrm{e} 0$ & $1.20 \mathrm{e}-2$ \\
\hline
\end{tabular}

(b) $N_{h}=196,608, N_{p}=65,536, N_{u}=131,072$.

the reduced bases using Algorithm 1 and constructing a DEIM approximation using Algorithm 3. The online stage involves computing the approximation to $\mathbb{E}[\vec{u}]$ and $\mathbb{E}[p]$. Results are presented in Table 6.3. The times for the online stage of the RB-DEIM-SCMFEM scheme are significantly quicker than the times for the high fidelity SCMFEM scheme, since the online cost does not depend on $N_{h}$. We also see that the total time (offline + online) for the RB-DEIM-SCMFEM scheme is quicker than for the high fidelity SCMFEM scheme since for a fixed $N_{h}, N_{R}$ does not increase significantly as $N_{l}$ increases.

Cost comparison with greedy RBM. Next, we compare the performance of our RBM, which uses Algorithm 1 to construct $Q_{u}$ and $Q_{p}$, with the standard greedy method. Algorithm 1 differs both in terms of the training set used and the method for selecting the points with which to form snapshots. In the standard greedy algorithm, randomly generated points are used, and in each iteration, the reduced bases are updated with the snapshot pair corresponding to the point $\mathbf{y} \in \Theta$ that maximises the estimated error $\Delta_{R}(\mathbf{y})$, continuing until $\Delta_{R}(\mathbf{y})<\epsilon_{1}$ for all $\mathbf{y} \in \Theta$. First, we select the same training set used in Algorithm 1 and perform the greedy algorithm on each sparse grid level. Next, we perform the greedy algorithm with $N_{l}$ randomly selected points. We use the DEIM approximation as before. In Table 6.4 we present the values of $N_{R}, K$, and the total time in seconds taken to perform the offline stage using the three approaches. We observe no more than a $10 \%$ difference in the values of $N_{R}$. However, the times recorded for Algorithm 1 are significantly lower than for the greedy approaches. The gains are substantial. This is because we only consider each point in the training set once, whereas the greedy approaches consider every point in each iteration. The results highlight the advantage of using a multilevel sparse grid approach, as more updates occur on the lower levels where there are fewer points. 
TABLE 6.3

Time in seconds for the RB-DEIM-SCMFEM scheme with $\epsilon_{1}=10^{-5}$, and the high fidelity SCMFEM scheme, to compute the expected solution for Example 6.1 on two spatial meshes as we increase $N_{l}$.

\begin{tabular}{|c|cc|cccc|c|}
\hline \multicolumn{9}{|c|}{} & \multicolumn{5}{|c|}{ RB-DEIM-SCMFEM } & SCMFEM \\
\hline$M$ & $l$ & $N_{l}$ & $N_{R}$ & $K$ & offline & online & high fidelity \\
\hline \multirow{4}{*}{8} & 3 & 849 & 43 & 84 & 28.8 & 1.7 & 43.0 \\
& 4 & 3937 & 44 & 83 & 111.0 & 7.3 & 198.7 \\
& 5 & 15,713 & 45 & 83 & 430.5 & 28.0 & 781.6 \\
& 6 & 56,737 & 46 & 83 & $1,553.6$ & 96.1 & 2873.6 \\
\hline \multirow{4}{*}{12} & 3 & 2,649 & 55 & 123 & 88.6 & 10.9 & 134.8 \\
& 4 & 17,265 & 59 & 124 & 538.9 & 76.8 & 875.8 \\
& 5 & 93,489 & 61 & 124 & $2,970.3$ & 447.3 & $4,777.2$ \\
\hline
\end{tabular}

(a) $N_{h}=12,288, N_{p}=4096, N_{u}=8192$.

\begin{tabular}{|c|cc|cccc|c|}
\hline \multicolumn{2}{|c|}{} & \multicolumn{4}{|c|}{ RB-DEIM-SCMFEM } & SCMFEM \\
\hline$M$ & $l$ & $N_{l}$ & $N_{R}$ & $K$ & offline & online & high fidelity \\
\hline \multirow{4}{*}{8} & 3 & 849 & 35 & 84 & 497.6 & 1.2 & $1,013.9$ \\
& 4 & 3,937 & 37 & 84 & 1895.0 & 5.8 & $4,685.2$ \\
& 5 & 15,713 & 40 & 84 & $7,352.9$ & 22.2 & $18,835.2$ \\
& 6 & 56,737 & 41 & 84 & $27,089.6$ & 80.5 & $68,305.7$ \\
\hline \multirow{4}{*}{12} & 3 & 2,649 & 52 & 124 & 1548.9 & 8.8 & $3,182.5$ \\
& 4 & 17,265 & 53 & 124 & $9,327.1$ & 57.6 & $20,746.1$ \\
& 5 & 93,489 & 53 & 124 & $53,685.8$ & 300.0 & $116,049.5$ \\
\hline
\end{tabular}

(b) $N_{h}=196,608, N_{p}=65,536, N_{u}=131,072$.

TABLE 6.4

Values of $N_{R}$ and $K$ and time in seconds for the offline stage where $Q_{u}$ and $Q_{p}$ are constructed using either Algorithm 1, the greedy algorithm with sparse grid points, or the greedy algorithm with random points for Example 6.1 with $M=8$ on two spatial meshes.

\begin{tabular}{|c|ccc|ccc|ccc|}
\hline & \multicolumn{3}{|c|}{ Algorithm 1 } & \multicolumn{3}{c|}{ Greedy (sparse grid) } & \multicolumn{3}{c|}{ Greedy (random) } \\
\hline$\epsilon_{1}$ & $N_{R}$ & $K$ & time (seconds) & $N_{R}$ & $K$ & time (seconds) & $N_{R}$ & $K$ & time (seconds) \\
\hline $1 \mathrm{e}-3$ & 10 & 38 & 77.0 & 12 & 38 & 80.6 & 14 & 38 & 959.4 \\
$1 \mathrm{e}-4$ & 26 & 60 & 93.1 & 27 & 60 & 203.5 & 25 & 60 & $1,891.9$ \\
$1 \mathrm{e}-5$ & 44 & 84 & 106.9 & 40 & 83 & 243.5 & 40 & 84 & $3,466.9$ \\
$1 \mathrm{e}-6$ & 67 & 114 & 157.7 & 66 & 114 & 986.1 & 63 & 113 & $6,414.7$ \\
\hline
\end{tabular}

(a) $N_{h}=12,288, N_{p}=4096, N_{u}=8192$.

\begin{tabular}{|c|ccc|ccc|ccc|}
\hline & \multicolumn{3}{|c|}{ Algorithm 1 } & \multicolumn{3}{|c|}{ Greedy (sparse grid) } & \multicolumn{3}{c|}{ Greedy (random) } \\
\hline$\epsilon_{1}$ & $N_{R}$ & $K$ & time (seconds) & $N_{R}$ & $K$ & time (seconds) & $N_{R}$ & $K$ & time (seconds) \\
\hline $1 \mathrm{e}-3$ & 10 & 38 & $1,171.7$ & 12 & 38 & $1,251.1$ & 10 & 38 & $11,485.3$ \\
$1 \mathrm{e}-4$ & 23 & 60 & $1,538.6$ & 23 & 60 & $2,131.9$ & 23 & 60 & $25,822.1$ \\
$1 \mathrm{e}-5$ & 37 & 83 & $1,887.1$ & 37 & 83 & $4,720.9$ & 36 & 84 & $51,806.8$ \\
$1 \mathrm{e}-6$ & 59 & 114 & $2,542.9$ & 55 & 113 & $7,109.5$ & 57 & 114 & $97,549.5$ \\
\hline
\end{tabular}

(b) $N_{h}=196,608, N_{p}=65,536, N_{u}=131,072$.

Issues affecting the size of the reduced bases. We have seen that the RB-DEIMSCMFEM scheme is efficient if $N_{R} \ll N_{h}$. Hence, a natural question is: what features of the 
problem affect $N_{R}$ ? Let $Z=V \times Q$ with $\|z\|_{Z}:=\|\vec{u}\|_{V}+\|p\|_{Q}$. For a fixed $\mathbf{y} \in \Gamma$, the high fidelity solution $z_{h}(\cdot, \mathbf{y})=\left(\vec{u}_{h}(\cdot, \mathbf{y}), p_{h}(\cdot, \mathbf{y})\right) \in Z_{h}=V_{h} \times Q_{h}$ satisfies (2.4) and the reduced solution $z_{R}(\cdot, \mathbf{y})=\left(\vec{u}_{R}(\cdot, \mathbf{y}), p_{R}(\cdot, \mathbf{y})\right) \in Z_{R}=V_{R} \times Q_{R}$ satisfies $(2.8)$. The quicker $\left\|z_{h}(\cdot, \mathbf{y})-z_{R}(\cdot, \mathbf{y})\right\|_{Z}$ decays to zero as $N_{R}$ increases, for all $\mathbf{y} \in \Gamma$ of interest, the smaller the value of $N_{R}$ we will need to ensure that the reduced basis error is smaller than a prescribed tolerance $\epsilon$. One way to obtain bounds for reduced basis errors is to seek bounds for the Kolmogorov width, see [39, Chapter 5]. For saddle point problems, see [16], this is defined as

$$
d_{N}(\Gamma)=\inf _{Z_{N} \subset Z_{h}, \operatorname{dim}}\left(\sup _{Z_{N}=N} \inf _{\mathbf{y} \in \Gamma}\left\|z_{N} \in Z_{N}(\cdot, \mathbf{y})-z_{N}(\cdot, \mathbf{y})\right\|_{Z_{h}}\right)
$$

For a fixed approximation space $Z_{N}$ of dimension $N$, the term in parentheses measures the worst case (in a point-wise sense on $\Gamma$ ) best approximation error. The Kolmogorov width characterises the smallest such error over all possible choices of $N$-dimensional spaces $Z_{N}$. The rate at which the Kolmogorov width decays with respect to $N$ then tells us the rate at which the reduced basis error decays, see $[8,15]$. It can be shown, see [16], that a bound for the Kolmogorov width is

$$
d_{N}(\Gamma) \leq C \sum_{k=1}^{M} \exp \left(-r_{k} N_{k}\right), \quad r_{k}=\log \left(\frac{2 \tau_{k}}{\left|\Gamma_{k}\right|}\left(1+\sqrt{1+\frac{\left|\Gamma_{k}\right|^{2}}{4 \tau_{k}^{2}}}\right)\right),
$$

where $\tau_{k}$ is the size of the region in the complex plane into which both $\vec{u}(\cdot, \mathbf{y})$ and $p(\cdot, \mathbf{y})$ admit an analytic extension with respect to $y_{k}$. The bound (6.4) exploits a result in [1, Theorem 4.1] for the error associated with a tensor product approximation to a scalar-valued function constructed on a grid with $N_{k}+1$ points in each direction so that $N=\prod_{k=1}^{M}\left(N_{k}+1\right)$. The authors in [7] consider the saddle point problem (3.5) posed on $\mathcal{D} \times \Gamma$, where $\Gamma=[-1,1]^{M}$ and $a_{M}^{-1}(\mathbf{x}, \mathbf{y})$ is a truncated Karhunen-Loève expansion. Assuming the mean and covariance of $a^{-1}(\mathbf{x}, \omega)$ are given, it is shown in [7, Lemma 4.1] that

$$
\tau_{k}:=\frac{a_{\min }}{\sqrt{\lambda_{k}}\left\|\phi_{k}(\mathbf{x})\right\|_{L^{\infty}(\mathcal{D})}}, \quad k=1, \ldots, M,
$$

where $\lambda_{k}, \phi_{k}(\mathbf{x})$ are eigenpairs of the covariance associated with $a^{-1}(\mathbf{x}, \omega)$. Combined with the bound in (6.4) this suggests that the quicker the terms $\lambda_{k}$ decay as $k \rightarrow \infty$, the smaller the space $Z_{N}$ needs to be to obtain an adequate reduced basis solution. This is what we observe for our parameter-dependent saddle point problem where we construct a reduced space $Z_{R}$ with dimension $N=3 N_{R}$. We now illustrate this.

Consider Example 6.1 with $M=8$. Again, we build the reduced bases using the training set $\Theta_{4}$, for which $\left|\Theta_{4}\right|=3,937$ (when $M=8$ ). In Table 6.5, we record the values of $N_{R}$ and $K$ returned by Algorithm 1 and Algorithm 3, respectively, as we increase the standard deviation $\sigma$ in (6.2) and vary the tolerance $\epsilon_{1}$. Note that $\sigma$ in (6.2) controls the magnitude of the $\lambda_{k}$ in (3.3). We observe that increasing $\sigma$, which increases $\lambda_{k}$, does indeed cause $N_{R}$ and $K$ to increase.

Next, we look at the effect that the rate of decay of the coefficients $\sqrt{\lambda_{k}}$ in (6.1) has on the values of $N_{R}$ returned by Algorithm 1 and the values of $K$ returned by Algorithm 3. We refer to $z_{M}(\mathbf{x}, \mathbf{y})$ from Example 6.1 as KL1, $z_{M}(\mathbf{x}, \mathbf{y})$ from Example 6.2 with $\tilde{\sigma}=2$ as KL2, $z_{M}(\mathbf{x}, \mathbf{y})$ from Example 6.2 with $\tilde{\sigma}=4$ as KL3, and $z_{M}(\mathbf{x}, \mathbf{y})$ from Example 6.3 as KL4. Note that in the KL1 case, $\sqrt{\lambda_{k}}=\mathcal{O}\left(k^{-1}\right)$, see [33, Example 7.58], and so these are the slowest decaying terms, while the values $\lambda_{k}$ in the KL4 case decay exponentially. These are the fastest decaying terms. Results are presented in Table 6.6 for several values of $\epsilon_{1}$. We observe that the KL1 case generally requires larger values of $N_{R}$ and $K$, while the KL4 case requires smaller values of $N_{R}$ and $K$.

We now examine the effect that the different rates of decay of the coefficients $\sqrt{\lambda_{k}}$ have on the convergence with respect to $N_{R}$ of the reduced basis error. First, we compute the maximum 
TABLE 6.5

Values of $N_{R}$ and $K$ required for Example 6.1 with $M=8$ on two spatial meshes, as we increase the standard deviation $\sigma$, with fixed training set $\Theta_{4}$.

\begin{tabular}{|c|cc|cc|cc|cc|}
\hline$\sigma$ & \multicolumn{2}{|c|}{0.1} & \multicolumn{2}{|c|}{0.25} & \multicolumn{2}{|c|}{0.5} & \multicolumn{2}{|c|}{1} \\
\hline$\epsilon_{1}$ & $N_{R}$ & $K$ & $N_{R}$ & $K$ & $N_{R}$ & $K$ & $N_{R}$ & $K$ \\
\hline $1 \mathrm{e}-3$ & 9 & 23 & 10 & 38 & 21 & 58 & 36 & 90 \\
$1 \mathrm{e}-4$ & 15 & 34 & 26 & 60 & 39 & 86 & 67 & 130 \\
$1 \mathrm{e}-5$ & 27 & 56 & 44 & 84 & 66 & 120 & 108 & 177 \\
$1 \mathrm{e}-6$ & 41 & 73 & 67 & 114 & 101 & 161 & 163 & 229 \\
\hline
\end{tabular}

(a) $N_{h}=12,288, N_{p}=4096, N_{u}=8192$.

\begin{tabular}{|c|cc|cc|cc|cc|}
\hline$\sigma$ & \multicolumn{2}{|c|}{0.1} & \multicolumn{2}{|c|}{0.25} & \multicolumn{2}{c|}{0.5} & \multicolumn{2}{|c|}{1} \\
\hline$\epsilon$ & $N_{R}$ & $K$ & $N_{R}$ & $K$ & $N_{R}$ & $K$ & $N_{R}$ & $K$ \\
\hline $1 \mathrm{e}-3$ & 8 & 23 & 10 & 38 & 19 & 58 & 29 & 90 \\
$1 \mathrm{e}-4$ & 11 & 34 & 23 & 60 & 34 & 86 & 55 & 130 \\
$1 \mathrm{e}-5$ & 25 & 56 & 37 & 84 & 56 & 120 & 92 & 178 \\
$1 \mathrm{e}-6$ & 37 & 73 & 59 & 114 & 87 & 161 & 146 & 230 \\
\hline
\end{tabular}

(b) $N_{h}=196,608, N_{p}=65,536, N_{u}=131,072$.

TABLE 6.6

Values of $N_{R}$ and $K$ required for Example 6.1 (KL1), Example 6.2 with $\tilde{\sigma}=2$ (KL2), Example 6.2 with $\tilde{\sigma}=4$ (KL3) and Example 6.3 (KL4) with $M=8$, on two spatial meshes with fixed training set $\Theta_{4}$.

\begin{tabular}{|c|cc|cc|cc|cc|}
\hline & \multicolumn{2}{|c|}{ KL1 } & \multicolumn{2}{c|}{ KL2 } & \multicolumn{2}{c|}{ KL3 } & \multicolumn{2}{c|}{ KL4 } \\
\hline$\epsilon_{1}$ & $N_{R}$ & $K$ & $N_{R}$ & $K$ & $N_{R}$ & $K$ & $N_{R}$ & $K$ \\
\hline $1 \mathrm{e}-3$ & 10 & 38 & 20 & 22 & 14 & 12 & 10 & 11 \\
$1 \mathrm{e}-4$ & 26 & 60 & 34 & 29 & 18 & 18 & 17 & 14 \\
$1 \mathrm{e}-5$ & 44 & 84 & 42 & 39 & 22 & 21 & 20 & 19 \\
$1 \mathrm{e}-6$ & 67 & 114 & 53 & 51 & 29 & 27 & 25 & 24 \\
\hline
\end{tabular}

(a) $N_{h}=12,288, N_{p}=4096, N_{u}=8192$.

\begin{tabular}{|c|cc|cc|cc|cc|}
\hline & \multicolumn{2}{|c|}{ KL1 } & \multicolumn{2}{c|}{ KL2 } & \multicolumn{2}{c|}{ KL3 } & \multicolumn{2}{c|}{ KL4 } \\
\hline$\epsilon_{1}$ & $N_{R}$ & $K$ & $N_{R}$ & $K$ & $N_{R}$ & $K$ & $N_{R}$ & $K$ \\
\hline $1 \mathrm{e}-3$ & 10 & 38 & 24 & 22 & 13 & 12 & 11 & 11 \\
$1 \mathrm{e}-4$ & 26 & 60 & 33 & 29 & 19 & 18 & 12 & 14 \\
$1 \mathrm{e}-5$ & 37 & 84 & 44 & 39 & 22 & 21 & 16 & 19 \\
$1 \mathrm{e}-6$ & 59 & 114 & 67 & 50 & 30 & 27 & 21 & 24 \\
\hline
\end{tabular}

(b) $N_{h}=196,608, N_{p}=65,536, N_{u}=131,072$.

relative pointwise error (over 100 randomly selected points $\mathbf{y} \in \Gamma$ ) between the reduced and high fidelity pressure and velocity approximations, which we denote by

$$
\delta_{R}^{p}=\max _{\mathbf{y} \in \Theta}\left(\frac{\left\|p_{h}(\cdot, \mathbf{y})-p_{R}(\cdot, \mathbf{y})\right\|_{Q}}{\left\|p_{h}(\cdot, \mathbf{y})\right\|_{Q}}\right), \quad \delta_{R}^{u}=\max _{\mathbf{y} \in \Theta}\left(\frac{\left\|\vec{u}_{h}(\cdot, \mathbf{y})-\vec{u}_{R}(\cdot, \mathbf{y})\right\|_{V}}{\left\|\vec{u}_{h}(\cdot, \mathbf{y})\right\|_{V}}\right) .
$$

In addition, we also compute the error between the high fidelity SCMFEM and RB-DEIMSCMFEM approximations of the mean of the pressure and the mean of the velocity, which we 


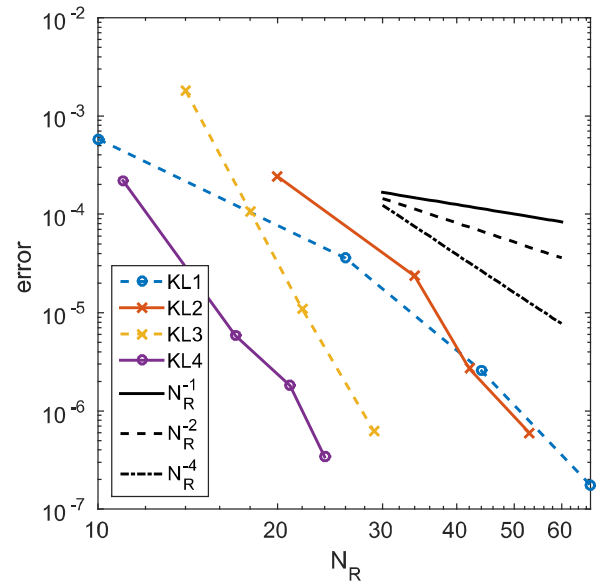

(a) Maximum pointwise pressure error $\left(\delta_{R}^{p}\right)$.

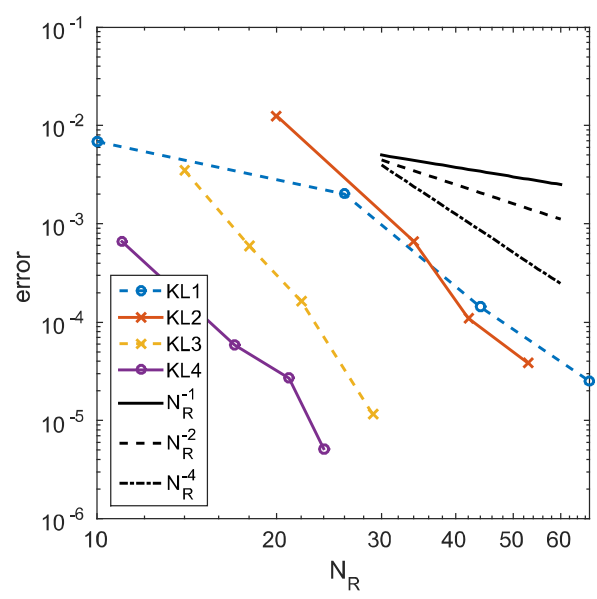

(c) Maximum pointwise velocity error $\left(\delta_{R}^{u}\right)$.

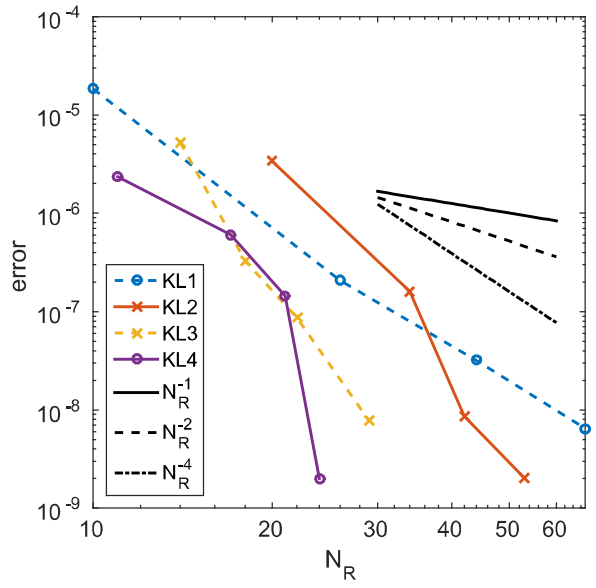

(b) Error in expectation of pressure $\left(\eta_{R}^{p}\right)$.

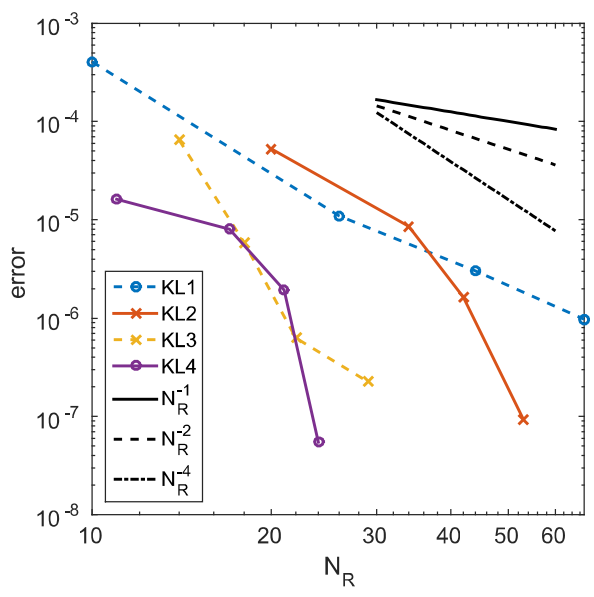

(d) Error in expectation of velocity $\left(\eta_{R}^{u}\right)$.

FIG. 6.1. Errors $\delta_{R}^{p}, \delta_{R}^{u}, \eta_{R}^{p}, \eta_{R}^{u}$ for Example 6.1 (KL1), Example 6.2 with $\tilde{\sigma}=2$ (KL2), with $\tilde{\sigma}=4$ (KL3) and Example 6.3 (KL4) with $M=8, N_{h}=12,288, N_{p}=4096, N_{u}=8192$ and training set $\Theta_{4}$ as we decrease $\epsilon_{1}$.

denote by

$$
\eta_{R}^{p}=\frac{\left\|\mathbb{E}\left[p_{R, \Theta}\right]-\mathbb{E}\left[p_{h, \Theta}\right]\right\|_{Q}}{\left\|\mathbb{E}\left[p_{h, \Theta}\right]\right\|_{Q}}, \quad \eta_{R}^{u}=\frac{\left\|\mathbb{E}\left[\vec{u}_{R, \Theta}\right]-\mathbb{E}\left[\vec{u}_{h, \Theta}\right]\right\|_{V}}{\left\|\mathbb{E}\left[\vec{u}_{h, \Theta}\right]\right\|_{V}} .
$$

We fix $N_{h}=12,288$ and use the training set $\Theta_{4}$ to construct the reduced bases. We present the errors $\delta_{R}^{p}, \delta_{R}^{u}, \eta_{R}^{p}, \eta_{R}^{u}$ in Figure 6.1 as we decrease $\epsilon_{1}$. For the KL1, KL2 and KL3 cases, we also indicate the decay rates of the $\sqrt{\lambda_{k}}$ terms for comparison. Each point on the curves in Figure 6.1 corresponds to $\epsilon_{1}=10^{-3}, 10^{-4}, 10^{-5}, 10^{-6}$, respectively. We observe that for the KL1 case, the errors $\delta_{R}^{p}, \delta_{R}^{u}, \eta_{R}^{p}, \eta_{R}^{u}$ decay the slowest, while for the KL4 case, the errors decay the fastest. We also observe that as $N_{R}$ increases, both the pointwise approximations and the RBDEIM-SCMFEM approximations of the mean converge. However, the velocity error $\delta_{R}^{u}$ is usually observed to be larger than $\epsilon_{1}$ while the pressure error $\delta_{R}^{p}$ is smaller. It appears that the reduced velocity approximation is converging slower than the reduced pressure approximation. This was also observed in [24] for a Stokes flow problem. 
Convergence comparison with greedy RBM. We now examine the decay of the reduced basis error incurred when $Q_{u}$ and $Q_{p}$ are constructed using Algorithm 1 as opposed to a standard greedy method. Note that theoretical bounds on the Kolmogorov width assume we use the latter. In Figure 6.2 we plot $\delta_{R}^{u}$ and $\delta_{R}^{p}$ as we increase $N_{R}$ for both Algorithm 1 and the greedy strategies described earlier. Similar rates of decay are observed for each approach.

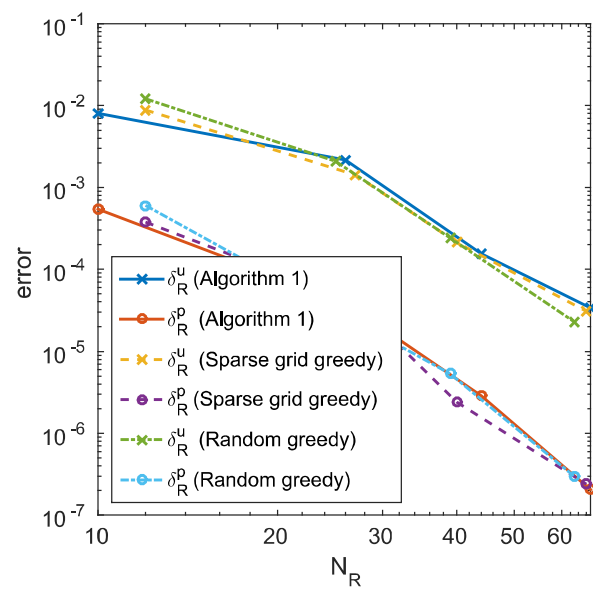

(a) $N_{h}=12,288, N_{p}=4096, N_{u}=8192$.

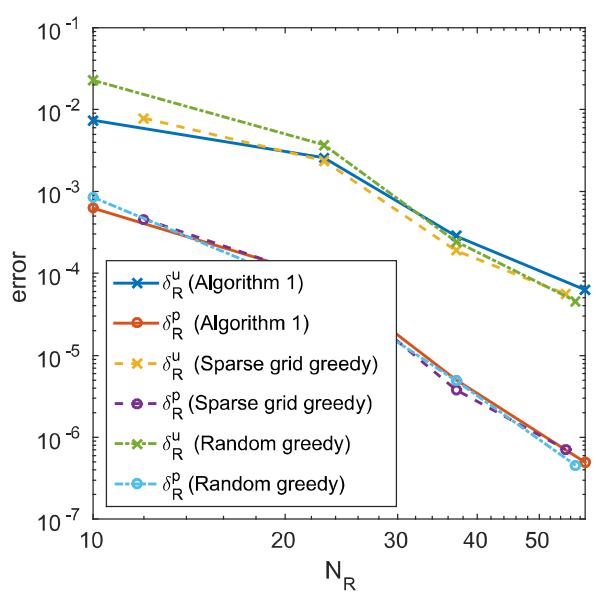

(b) $N_{h}=196,608, N_{p}=65,536, N_{u}=131,072$.

FIG. 6.2. Errors $\delta_{R}^{u}$ and $\delta_{R}^{p}$ for Example 6.1 with $M=8$ as $\epsilon_{1} \rightarrow 0$, where $Q_{u}$ and $Q_{p}$ are constructed using Algorithm 1 with training set $\Theta_{4}$, the greedy algorithm with training set $\Theta_{4}$, or the greedy algorithm with $N_{4}=\left|\Theta_{4}\right|$ randomly generated samples.

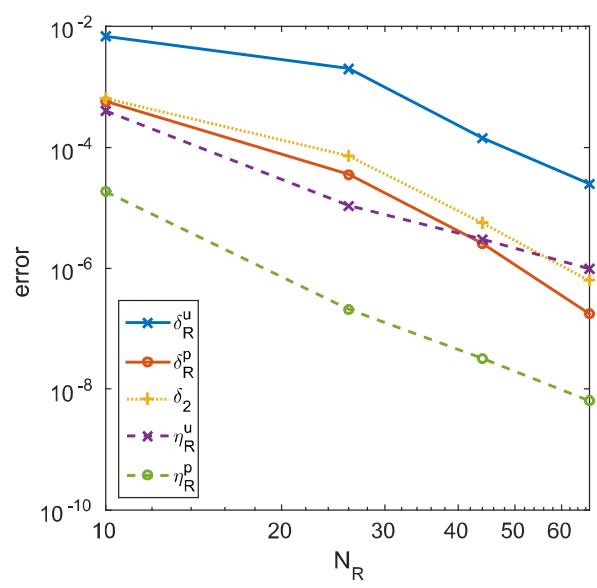

(a) Errors $\delta_{R}^{p}, \eta_{R}^{p}, \delta_{R}^{u}, \eta_{R}^{u}$, and residual $\delta_{2}$.

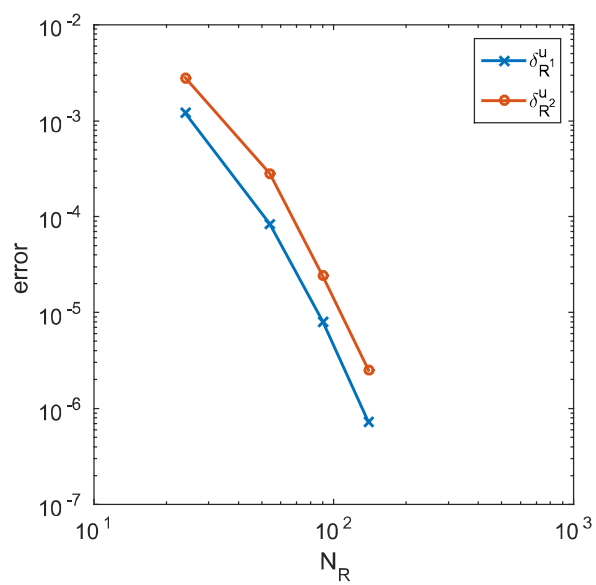

(b) Errors $\delta_{R}^{u_{1}}$ and $\delta_{R}^{u_{2}}$.

FIG. 6.3. (a) Errors $\delta_{R}^{p}, \eta_{R}^{p}, \delta_{R}^{u}, \eta_{R}^{u}$, and maximum relative residual $\delta_{2}$ for Example 6.1 (KL1) with $M=8$ and $f=0$, (b) velocity component errors $\delta_{R}^{u_{1}}$ and $\delta_{R}^{u_{2}}$ for Example 6.1 with $M=12$ and $f\left(x_{1}, x_{2}\right)=2\left(x_{1}+x_{2}-x_{1}^{2}-x_{2}^{2}\right)$. In both experiments, $N_{h}=12,288, N_{p}=4096, N_{u}=8192$.

Unlike the standard greedy RBM, Algorithm 1 uses the discrete relative residual error as an error indicator. In Figure 6.3a we plot the errors $\delta_{R}^{p}, \eta_{R}^{p}, \delta_{R}^{u}$ and $\eta_{R}^{u}$ for Example 6.1 (the KL1 
case) again, as well as $\delta_{2}$, as we vary the tolerance $\epsilon_{1}$. Recall that we have $\delta_{2} \approx \delta_{1} \leq \epsilon_{1}$. We observe that $\delta_{R}^{p}, \delta_{R}^{u}$ and $\delta_{2}$ decay at a similar rate, although $\delta_{R}^{u}$ is larger than $\delta_{2}$. However, for the expectation, $\eta_{R}^{u}$ is close to $\delta_{2}$. While it is not certified in the usual sense, our RBM does a good enough job in delivering estimates of the quantities of interest in this problem.

Coercivity. Finally, recall that it is desirable to have coercivity with respect to $\|\cdot\|_{\mathrm{H}(\mathrm{div}, \mathcal{D})}$ so that we obtain a priori error estimates for the velocity error using standard theory. Here, $\|\vec{v}\|_{V}^{2}=\|\vec{v}\|_{L^{2}(D)}^{2}+\|\nabla \cdot \vec{v}\|_{L^{2}(D)}^{2}$. For a given $\mathbf{y} \in \Gamma$ let

$$
\delta_{R}^{u_{1}}(\mathbf{y})=\frac{\left\|\vec{u}_{h}(\cdot, \mathbf{y})-\vec{u}_{R}(\cdot, \mathbf{y})\right\|_{L^{2}(\mathcal{D})}}{\left\|\vec{u}_{h}(\cdot, \mathbf{y})\right\|_{L^{2}(\mathcal{D})}}, \quad \delta_{R}^{u_{2}}(\mathbf{y})=\frac{\left\|\nabla \cdot\left(\vec{u}_{h}(\cdot, \mathbf{y})-\vec{u}_{R}(\cdot, \mathbf{y})\right)\right\|_{L^{2}(\mathcal{D})}}{\left\|\nabla \cdot\left(\vec{u}_{h}(\cdot, \mathbf{y})\right)\right\|_{L^{2}(\mathcal{D})}}
$$

If both $\delta_{R}^{u_{1}}(\mathbf{y})$ and $\delta_{R}^{u_{2}}(\mathbf{y})$ decay at the same rate as $N_{R}$ increases, this suggests that the velocity approximation is stable with respect to the $\mathrm{H}(\operatorname{div}, \mathcal{D})$ norm. In Figure $6.3 \mathrm{~b}$, we plot $\delta_{R}^{u_{1}}(\mathbf{y})$ and $\delta_{R}^{u_{2}}(\mathbf{y})$ for a single representative $\mathbf{y} \in \Gamma$ for a test problem and observe that this is the case.

6.2. Groundwater flow test problem. Finally, we consider a test problem of the form (3.5) with $f(\mathbf{x})=0$ from the software package PIFISS [44]. Let the spatial domain $\mathcal{D}=\cup_{k=1}^{5} D_{k}$ be made up of 5 non-overlapping subdomians $D_{k}$, as depicted in Figure 6.4, which is based on the geometry of a real underground location, see [40]. On the boundary, we set $p=1$ on the left edge (the inflow), $p=0$ on the right edge (the outflow), and choose homogeneous Neumann conditions for the velocity on all other edges. We use the MATLAB PDE toolbox to construct two non-uniform spatial meshes. For the coefficient, let $\left.a_{M}(\mathbf{x}, \mathbf{y})\right|_{D_{k}}=y_{k}, i=1, \ldots, 5$ so realisations of $a_{M}(\mathbf{x}, \mathbf{y})$ are piecewise constant in each subdomain $D_{k}$. For the high fidelity approximation, for each $\mathbf{y} \in \Gamma$, we apply lowest-order triangular Raviart-Thomas elements. The snapshot pair $\left(\vec{u}_{h}(\cdot, \mathbf{y}), p_{h}(\cdot, \mathbf{y})\right)$ corresponding to the specific choice of parameters $\mathbf{y}=[1.04,17.2,0.31,2.60,17.2]^{\top}$, which comes from the deterministic groundwater flow model described in [40], is plotted in Figure 6.5.

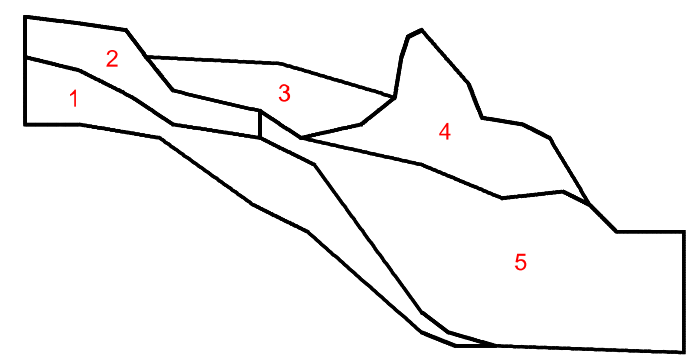

FIG. 6.4. Computational domain $\mathcal{D}$ for the groundwater flow test problem.

Now, we treat the coefficients as uncertain, and choose $\left.a_{M}(\mathbf{x}, \mathbf{y})\right|_{D_{k}}=\exp \left(y_{k}\right)$ for $k=1, \ldots, 5$, where each $y_{k}$ is the image of a Gaussian random variable $\xi_{k} \sim N\left(\mu_{k}, \sigma_{k}\right)$, with mean $\mu_{k}$ and standard deviation $\sigma_{k}$ chosen so that

$$
\begin{aligned}
& \mathbb{E}\left[\exp \left(\xi_{1}\right)\right]=1.04, \quad \operatorname{Var}\left(\exp \left(\xi_{1}\right)\right)=0.832, \\
& \mathbb{E}\left[\exp \left(\xi_{2}\right)\right]=17.2, \quad \operatorname{Var}\left(\exp \left(\xi_{2}\right)\right)=13.76, \\
& \mathbb{E}\left[\exp \left(\xi_{3}\right)\right]=0.31, \quad \operatorname{Var}\left(\exp \left(\xi_{3}\right)\right)=0.248, \\
& \mathbb{E}\left[\exp \left(\xi_{4}\right)\right]=2.60, \quad \operatorname{Var}\left(\exp \left(\xi_{4}\right)\right)=2.08, \\
& \mathbb{E}\left[\exp \left(\xi_{5}\right)\right]=17.2, \quad \operatorname{Var}\left(\exp \left(\xi_{5}\right)\right)=13.76 .
\end{aligned}
$$




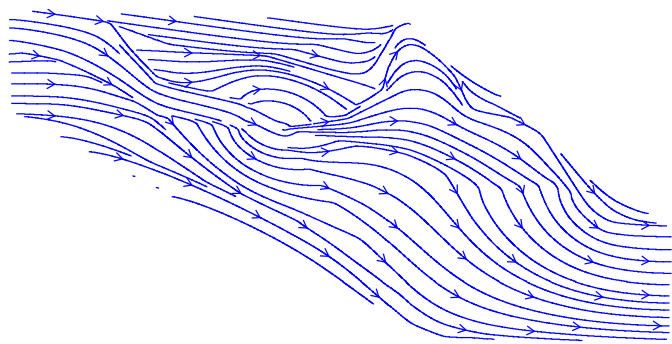

(a) velocity streamlines

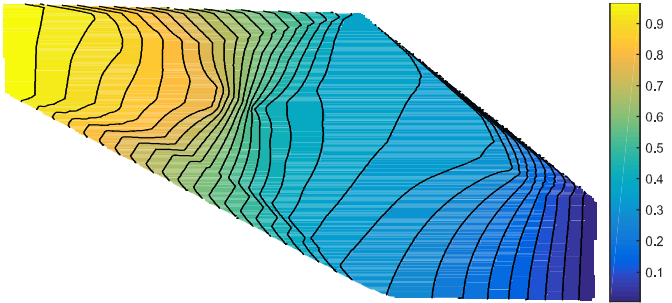

(b) pressure

FIG. 6.5. High fidelity approximation to the groundwater flow problem with $N_{h}=13,686, N_{p}=5424$, $N_{u}=8262$ corresponding to the inputs $y_{1}=1.04, y_{2}=17.2, y_{3}=0.31, y_{4}=2.60, y_{5}=17.2$.

That is, on each subdomain $D_{k}, a_{M}$ is a lognormal random variable. Then, we have

$$
a_{M}(\mathbf{x}, \mathbf{y})^{-1}=\sum_{k=1}^{5} \exp \left(-y_{k}\right) 1_{D_{k}}(\mathbf{x}) .
$$

This is an affine function of $M=K=5$ parameters and so we do not need to perform DEIM. We construct RB matrices $Q_{u}$ and $Q_{p}$ using Algorithm 1 with tolerance $\epsilon_{1}$ and the training set $\Theta_{l}$ is chosen to be a sparse grid based on Gauss points. Note that Gauss points are not nested. When we update the RB matrices $Q_{u}$ and $Q_{p}$ at each iteration of Algorithm 1 we also compute the offline matrices and vectors in (4.7) and the quantities needed to evaluate the error indicator (4.3). Since $K$ is small here, we find that this is the cheapest offline strategy. In Table 6.7, we record the average time taken to assemble and solve the high fidelity system (3.10) and the reduced system (3.19) as we decrease the tolerance $\epsilon_{1}$. In all cases we see that $N_{R} \ll N_{h}$ and so the cost of solving (3.19) is substantially cheaper than solving (3.10).

TABLE 6.7

Average time in seconds to assemble and solve the high fidelity systems (3.10) (T) and the RB systems (3.19) $(t)$ over 100 points $\mathbf{y} \in \Gamma$ for the groundwater flow problem on two spatial meshes with training set $\Theta_{4}$.

\begin{tabular}{|ccc|cccc|}
\hline$N_{h}$ & $N_{p}$ & $N_{u}$ & $\epsilon_{1}$ & $N_{R}$ & $T$ & $t$ \\
\hline \multirow{4}{*}{13,686} & \multirow{3}{*}{5424} & \multirow{2}{*}{8262} & $1 \mathrm{e}-3$ & 39 & $7.32 \mathrm{e}-2$ & $6.64 \mathrm{e}-4$ \\
& & & $1 \mathrm{e}-4$ & 60 & $6.93 \mathrm{e}-2$ & $1.04 \mathrm{e}-3$ \\
& & & $1 \mathrm{e}-5$ & 82 & $6.87 \mathrm{e}-2$ & $1.61 \mathrm{e}-3$ \\
& & & $1 \mathrm{e}-3$ & 36 & $6.70 \mathrm{e}-2$ & $2.56 \mathrm{e}-3$ \\
\hline \multirow{4}{*}{217,464} & \multirow{3}{*}{86,784} & \multirow{2}{*}{130,680} & $1 \mathrm{e}-4$ & 59 & $2.14 \mathrm{e} 0$ & $5.57 \mathrm{e}-4$ \\
& & & $1 \mathrm{e}-5$ & 84 & $2.14 \mathrm{e} 0$ & $1.06 \mathrm{e}-3$ \\
& & & $1 \mathrm{e}-6$ & 108 & $2.08 \mathrm{e} 0$ & $5.03 \mathrm{e}-3$ \\
\hline
\end{tabular}

Finally, we compare the time taken to estimate $\mathbb{E}[\vec{u}]$ and $\mathbb{E}[p]$ using the RB-SCMFEM approximation and the high fidelity SCMFEM approximation, as we increase the number of collocation points $N_{l}=\left|\Theta_{l}\right|$. The offline stage of the RB-SCMFEM scheme involves constructing the reduced matrices $Q_{u}$ and $Q_{p}$, and the online stage involves computing the approximation to $\mathbb{E}[\vec{u}]$ and $\mathbb{E}[p]$. The results are presented in Table 6.8. Since Gaussian random variables are unbounded, the set of sparse grid points $\Theta_{l}$ is also unbounded as we increase the level number $l$. We see that $N_{R}$ increases as we increase $l$. In all cases, however, $N_{R}$ is small enough that applying the RB-SCMFEM scheme is significantly cheaper than applying the high fidelity SCMFEM scheme. In particular, 
TABLE 6.8

Time in seconds for the RB-SCMFEM scheme and high fidelity SCMFEM scheme for the groundwater flow problem on two spatial meshes with $\epsilon_{1}=10^{-5}$ as we increase $N_{l}$.

\begin{tabular}{|c|c|c|c|c|c|c|c|c|}
\hline & & & & & \multicolumn{3}{|c|}{ RB-SCMFEM } & SCMFEM \\
\hline$N_{h}$ & $\overline{N_{p}}$ & $N_{u}$ & $\bar{l}$ & $N_{l}$ & $N_{R}$ & offline & online & high fidelity \\
\hline \multirow{4}{*}{13,686} & \multirow{4}{*}{5424} & \multirow{4}{*}{8262} & 3 & 351 & 64 & 23.0 & 0.4 & 24.0 \\
\hline & & & 4 & 1,471 & 82 & 40.2 & 2.4 & 98.0 \\
\hline & & & 5 & 5,503 & 95 & 75.6 & 12.0 & 368.3 \\
\hline & & & 6 & 18,943 & 103 & 183.9 & 48.8 & $1,292.0$ \\
\hline \multirow{3}{*}{217,464} & \multirow{3}{*}{86,784} & \multirow{3}{*}{130,680} & 3 & 351 & 64 & 510.9 & 0.4 & 746.0 \\
\hline & & & 4 & 1,471 & 84 & 795.0 & 2.6 & $3,058.0$ \\
\hline & & & 5 & 5,503 & 96 & $1,002.1$ & 14.3 & $11,523.5$ \\
\hline
\end{tabular}

from the last line of Table 6.8 we see that the high fidelity SCMFEM scheme is over 10 times as expensive as our reduced basis method.

7. Concluding remarks. In this paper we described the application of reduced basis methods to parameter-dependent saddle point problems of the form (1.1) and developed an efficient method for performing forward UQ for the groundwater flow problem (3.1). We presented a non-standard training algorithm for constructing a pair of compatible reduced bases that uses a multilevel approach based on sparse grid points, and a simple error indicator. We also described how to combine this with a DEIM approximation to the inverse of the diffusion coefficient. We demonstrated that with these variations we gain substantially lower offline times when compared to the standard greedy algorithm. We showed that assembling and solving the RB-DEIM system (3.21) is much cheaper than assembling and solving the high fidelity system (3.10). We combined the reduced basis method with a stochastic collocation mixed finite element method and implemented the resulting RB-DEIM-SCMFEM scheme for a range of test problems, demonstrating that significant computational savings can be made over standard high fidelity SCMFEM schemes, even when the offline times are taken into account. Our experiments reveal, however, that reduced basis methods are most effective for performing forward UQ for PDE models with quickly decaying coefficient terms, a modest number of parameters and low variances.

\section{REFERENCES}

[1] I. BAbušKa, F. Nobile, And R. Tempone, A stochastic collocation method for elliptic partial differential equations with random input data, SIAM Journal on Numerical Analysis, 45 (2007), pp. 1005-1034, doi:10.1137/050645142.

[2] I. BAbušKa, R. Tempone, AND G. E. Zouraris, Galerkin finite element approximations of stochastic elliptic partial differential equations, SIAM Journal on Numerical Analysis, 42 (2004), pp. 800-825, doi:10.1137/S0036142902418680.

[3] M. Barrault, Y. Maday, N. C. Nguyen, and A. T. Patera, An empirical interpolationmethod: application to efficient reduced-basis discretization of partial differential equations, Comptes Rendus Mathematique, 339 (2004), pp. 667-672, doi:10.1016/j.crma.2004.08.006.

[4] V. Barthelmann, E. NovaK, And K. Ritter, High dimensional polynomial interpolation on sparse grids, Advances in Computational Mathematics, 12 (2000), pp. 273-288, doi:10.1023/A:1018977404843.

[5] P. Benner AND M. W. Hess, Reduced basis modeling for uncertainty quantification of electromagnetic problems in stochastically varying domains, in Scientific Computing in Electrical Engineering, Springer, 2016, pp. 215-222.

[6] M. Benzi, G. H. Golub, And J. Liesen, Numerical solution of saddle point problems, Acta numerica, 14 (2005), pp. 1-137, doi:10.1017/S0962492904000212.

[7] A. Bespalov, C. E. Powell, and D. J. Silvester, A priori error analysis of stochastic Galerkin mixed approximations of elliptic pdes with random data, SIAM Journal on Numerical Analysis, 50 (2012), pp. 2039-2063. 
[8] P. Binev, A. Cohen, W. Dahmen, R. DeVore, G. Petrova, and P. Wojtaszczyk, Convergence rates for greedy algorithms in reduced basis methods, SIAM journal on mathematical analysis, 43 (2011), pp. 14571472 .

[9] D. Boffi, F. Brezzi, And M. Fortin, Mixed finite element methods and applications, Springer, 2013.

[10] S. Boyaval, C. Le Bris, T. Lelivre, Y. Maday, N. Nguyen, and A. Patera, Reduced basis techniques for stochastic problems, Archives of Computational Methods in Engineering, 17 (2010), pp. 435-454, doi:10.1007/s11831-010-9056-z.

[11] S. C. Brenner And R. Scott, The mathematical theory of finite element methods, vol. 15, Springer Science \& Business Media, 2008.

[12] S. Chaturantabut and D. C. Sorensen, Nonlinear model reduction via discrete empirical interpolation, SIAM Journal on Scientific Computing, 32 (2010), pp. 2737-2764.

[13] P. Chen AND A. Quarteroni, A new algorithm for high-dimensional uncertainty quantification based on dimension-adaptive sparse grid approximation and reduced basis methods, Journal of Computational Physics, 298 (2015), pp. 176-193.

[14] P. Chen, A. Quarteroni, And G. Rozza, A weighted reduced basis method for elliptic partial differential equations with random input data, SIAM Journal on Numerical Analysis, 51 (2013), pp. 3163-3185.

[15] P. Chen, A. Quarteroni, And G. RozzA, Comparison between reduced basis and stochastic collocation methods for elliptic problems, Journal of Scientific Computing, 59 (2014), pp. 187-216, doi:10.1007/s10915013-9764-2.

[16] P. Chen, A. Quarteroni, And G. Rozza, Multilevel and weighted reduced basis method for stochastic optimal control problems constrained by Stokes equations, Numerische Mathematik, 133 (2016), pp. 67-102.

[17] P. Chen and C. Schwab, Sparse-grid, reduced-basis bayesian inversion, Computer Methods in Applied Mechanics and Engineering, 297 (2015), pp. 84-115.

[18] K. Cliffe, I. G. Graham, R. Scheichl, and L. Stals, Parallel computation of flow in heterogeneous media modelled by mixed finite elements, Journal of Computational Physics, 164 (2000), pp. 258-282.

[19] M. Eigel, C. J. Gittelson, C. Schwab, And E. Zander, Adaptive stochastic Galerkin FEM, Computer Methods in Applied Mechanics and Engineering, 270 (2014), pp. 247-269.

[20] H. C. Elman and V. Forstall, Preconditioning techniques for reduced basis methods for parameterized elliptic partial differential equations, SIAM Journal on Scientific Computing, 37 (2015), pp. S177-S194, doi:10.1137/140970859.

[21] H. C. Elman and V. Forstald, Numerical solution of the steady-state Navier-Stokes equations using empirical interpolation methods, arXiv preprint arXiv:1605.06138, (2016).

[22] H. C. ElmAN AND Q. LiAO, Reduced basis collocation methods for partial differential equations with random coefficients, SIAM/ASA Journal on Uncertainty Quantification, 1 (2013), pp. 192-217, doi: $10.1137 / 120881841$.

[23] B. Ganis, H. Klie, M. F. Wheeler, T. Wildey, I. Yotov, And D. Zhang, Stochastic collocation and mixed finite elements for flow in porous media, Computer methods in applied mechanics and engineering, 197 (2008), pp. 3547-3559.

[24] A.-L. Gerner And K. Veroy, Certified reduced basis methods for parametrized saddle point problems, SIAM Journal on Scientific Computing, 34 (2012), pp. A2812-A2836.

[25] A. D. Gordon And C. E. Powell, On solving stochastic collocation systems with algebraic multigrid, IMA Journal of Numerical Analysis, (2011).

[26] I. G. Graham, R. Scheichl, and E. Ullmann, Mixed finite element analysis of lognormal diffusion and multilevel monte carlo methods, Stochastics and Partial Differential Equations Analysis and Computations, 4 (2016), pp. 41-75.

[27] A. GREenbaum, Iterative methods for solving linear systems, vol. 17, SIAM, 1997.

[28] M. A. Grepl, Y. Maday, N. C. NGuyen, and A. T. Patera, Efficient reduced-basis treatment of nonaffine and nonlinear partial differential equations, ESAIM: Mathematical Modelling and Numerical Analysis, 41 (2007), pp. 575-605.

[29] M. D. Gunzburger, C. G. Webster, and G. Zhang, Stochastic finite element methods for partial differential equations with random input data, Acta Numerica, 23 (2014), pp. 521-650, doi:10.1017/S0962492914000075.

[30] B. HaAsdonk, K. Urban, And B. Wieland, Reduced basis methods for parameterized partial differential equations with stochastic influences using the karhunen-loeve expansion, SIAM/ASA Journal on Uncertainty Quantification, 1 (2013), pp. 79-105.

[31] J. S. Hesthaven, G. Rozza, and B. Stamm, Certified reduced basis methods for parametrized partial differential equations, SpringerBriefs in Mathematics, (2015).

[32] Q. LiaO AND G. Lin, Reduced basis ANOVA methods for partial differential equations with high-dimensional random inputs, Journal of Computational Physics, 317 (2016), pp. 148-164.

[33] G. J. Lord, C. E. Powell, And T. Shardlow, An introduction to computational stochastic PDEs, Cambridge University Press, 2014.

[34] I. Martini, G. RozZA, AND B. HAASDONK, Reduced basis approximation and a-posteriori error estimation for 
the coupled Stokes-Darcy system, Advances in Computational Mathematics, 41 (2015), pp. 1131-1157.

[35] F. Nobile, R. Tempone, And C. G. Webster, A sparse grid stochastic collocation method for partial differential equations with random input data, SIAM Journal on Numerical Analysis, 46 (2008), pp. 2309-2345, doi:10.1137/060663660, arXiv:http://dx.doi.org/10.1137/060663660.

[36] C. C. Paige and M. A. Saunders, Solution of sparse indefinite systems of linear equations, SIAM journal on numerical analysis, 12 (1975), pp. 617-629.

[37] C. E. Powell, D. Silvester, And V. Simoncini, An efficient reduced basis solver for stochastic Galerkin matrix equations, Preprint, (2015), http://eprints.ma.man.ac.uk/2350/01/Powell_Silvester_Simoncini.pdf.

[38] C. E. Powell and D. J. Silvester, Optimal preconditioning for Raviart-Thomas mixed formulation of second-order elliptic problems, SIAM journal on matrix analysis and applications, 25 (2003), pp. 718-738.

[39] A. Quarteroni, A. Manzoni, AND F. Negri, Reduced basis methods for partial differential equations: an introduction, Springer International Publishing, 2016.

[40] B. Riviè, M. F. Wheeler, and C. Baumann, Part II. discontinuous Galerkin method applied to a single phase flow in porous media. https://www.ices.utexas.edu/media/reports/1999/9910.pdf, 1999.

[41] G. RozzA, D. B. P. Huynh, And A. Manzoni, Reduced basis approximation and a posteriori error estimation for Stokes flows in parametrized geometries: roles of the inf-sup stability constants, Numerische Mathematik, 125 (2013), pp. 115-152.

[42] G. Rozza And K. Veroy, On the stability of the reduced basis method for Stokes equations in parametrized domains, Computer methods in applied mechanics and engineering, 196 (2007), pp. 1244-1260.

[43] T. Rusten And R. Winther, A preconditioned iterative method for saddlepoint problems, SIAM Journal on Matrix Analysis and Applications, 13 (1992), pp. 887-904.

[44] D. J. Silvester And C. E. Powell, Potential Incompressible Flow and Iterative Solver Software (PIFISS) version 1.0, February 2007. http://www.maths.manchester.ac.uk/ djs/ifiss/pifiss.html.

[45] R. C. SмIтH, Uncertainty quantification: theory, implementation, and applications, vol. 12, SIAM, 2013.

[46] S. A. Smolyak, Quadrature and interpolation formulas for tensor products of certain classes of functions, Dokl. Akad. Nauk SSSR, 4 (1963), p. 123.

[47] A. M. STUART, Inverse problems: a Bayesian perspective, Acta Numer., 19 (2010), pp. 451-559, doi:10.1017/S0962492910000061.

[48] T. J. Sullivan, Introduction to Uncertainty Quantification, vol. 63, Springer, 2016.

[49] L. N. Trefethen And D. BAu III, Numerical linear algebra, vol. 50, SIAM, 1997.

[50] D. XiU And J. S. Hesthaven, High-order collocation methods for differential equations with random inputs, SIAM Journal on Scientific Computing, 27 (2005), pp. 1118-1139, doi:10.1137/040615201. 